\title{
Mordell integrals and Giveon-Kutasov duality
}

\author{
Georgios Giasemidis ${ }^{a}$ and Miguel Tierz ${ }^{b, c}$ \\ ${ }^{a}$ CountingLab LTD \& Centre for the Mathematics of Human Behaviour (CMoHB), \\ Department of Mathematics and Statistics, University of Reading, Reading, RG6 6AX U.K. \\ ${ }^{b}$ Departamento de Matemática, Grupo de Física Matemática, Faculdade de Ciências, \\ Universidade de Lisboa, Campo Grande, Edifício C6, Lisboa, 1749-016 Portugal \\ ${ }^{c}$ Departamento de Análisis Matemático, Facultad de Ciencias Matemáticas, \\ Universidad Complutense de Madrid, Madrid, 28040 Spain \\ E-mail: g.giasemidis@reading.ac.uk, tierz@fc.ul.pt
}

Abstract: We solve, for finite $N$, the matrix model of supersymmetric $\mathrm{U}(N)$ ChernSimons theory coupled to $N_{\mathrm{f}}$ massive hypermultiplets of $R$-charge $\frac{1}{2}$, together with a FayetIliopoulos term. We compute the partition function by identifying it with a determinant of a Hankel matrix, whose entries are parametric derivatives (of order $N_{\mathrm{f}}-1$ ) of Mordell integrals. We obtain finite Gauss sums expressions for the partition functions. We also apply these results to obtain an exhaustive test of Giveon-Kutasov (GK) duality in the $\mathcal{N}=3$ setting, by systematic computation of the matrix models involved. The phase factor that arises in the duality is then obtained explicitly. We give an expression characterized by modular arithmetic ( $\bmod 4)$ behavior that holds for all tested values of the parameters (checked up to $N_{\mathrm{f}}=12$ flavours).

Keywords: Matrix Models, Duality in Gauge Field Theories, Supersymmetric gauge theory, Chern-Simons Theories

ArXIV EPRINT: 1511.00203 


\section{Contents}

1 Introduction 1

2 Parametric derivatives of Mordell integrals for the arbitrary $N_{\mathrm{f}}$ setting 4

2.1 The theory with $2 N_{\mathrm{f}}$ hypermultiplets 6

3 Analytical expressions for the partition functions and some interpretations

3.1 Abelian partition functions 8

$\begin{array}{lll}3.2 \mathrm{U}(2) \text { gauge group } & 11\end{array}$

3.3 Supersymmetry breaking and partition functions of modulus $1 \quad 12$

$\begin{array}{lll}3.4 & \mathrm{U}(3) \text { gauge group } & 12\end{array}$

$\begin{array}{ll}3.5 \text { Cases with } N_{\mathrm{f}}=3 & 13\end{array}$

4 Giveon-Kutasov duality $\quad 14$

$\begin{array}{lll}4.1 & \text { Explicit expression for the phase factor } & 15\end{array}$

$\begin{array}{lll}4.2 & \text { Massive hypemultiplets and non-zero Fayet-Iliopoulos term } & 18\end{array}$

$\begin{array}{lll}4.3 & N_{\mathrm{f}} \text { hypermultiplets with mass } m \text { and } N_{\mathrm{f}} \text { hypermultiplets with mass }-m & 19\end{array}$

$4.4 N_{\mathrm{f}}$ hypermultiplets with mass $m_{1}$ and $N_{\mathrm{f}}$ hypermultiplets with mass $m_{2} \quad 20$

\section{Introduction}

The study of supersymmetric gauge theories has greatly benefited in recent years from the development of the localization of supersymmetric gauge theories by Pestun [1] ([2-4] for recent reviews). The localization procedure manages to reduce the original functional integral describing a quantum field theory into a much simpler matrix integral. Thus, it enormously reduces the task of computing observables in a supersymmetric gauge theory. However, there still remains the issue of explicitly computing $N$ integrations, in which case one needs to employ matrix model tools [5] in order to obtain explicit expressions for the observables of the gauge theory.

The theory we shall focus on is $\mathcal{N}=2$ and $\mathcal{N}=3$ supersymmetric $\mathrm{U}(N)$ Chern-Simons (CS) on three-sphere, $\mathbb{S}^{3}$, with $N_{\mathrm{f}}$ fundamental and $N_{\mathrm{f}}$ anti-fundamental chiral multiplets of mass $m$. Indeed the partition function on $\mathbb{S}^{3}$ can be determined by the localization techniques of [1], which were used in the $3 \mathrm{~d}$ case in [6-9]. In the case of the partition function for $\mathrm{U}(N) \mathcal{N}=2$ Chern-Simons theory at level $k$ coupled to $N_{\mathrm{f}}$ fundamental and 
$\bar{N}_{\mathrm{f}}$ anti-fundamental chiral multiplets of $R$-charge $q$ the matrix model is $[9]^{1}$

$Z=\frac{1}{N !} \int d^{N} \sigma \prod_{j=1}^{N} e^{\mathrm{i} \pi k \sigma_{j}^{2}}\left(s_{b=1}\left(\mathrm{i}-\mathrm{i} q-\sigma_{j}\right)\right)^{N_{\mathrm{f}}}\left(s_{b=1}\left(\mathrm{i}-\mathrm{i} q+\sigma_{j}\right)\right)^{\bar{N}_{f}} \prod_{i<j}^{N}\left(2 \sinh \pi\left(\sigma_{i}-\sigma_{j}\right)\right)^{2}$,

where $s_{b=1}(\sigma)$ denotes the double sine function $[8,9]$ (and references therein). This matrix model corresponds to the case where the matter chiral multiplets have $R$-charge $q$ and belong to the representation $R$ of the gauge group. The fact that for $\mathcal{N}=3$ theories the $\mathrm{R}$-symmetry is non-abelian allows us to fix an $R$-charge which is not altered under the RG flow. In this paper, we focus on a detailed study of the case where $q=1 / 2$ and $R=r \oplus \bar{r}$. In this case, due to the basic property of the double sine function [9]

$$
\prod_{\rho \in r} s_{b=1}\left(\frac{\mathrm{i}}{2}-\rho_{i} \hat{\sigma}_{i}\right) \cdot s_{b=1}\left(\frac{\mathrm{i}}{2}+\rho_{i} \hat{\sigma}_{i}\right)=\prod_{\rho \in r} \frac{1}{2 \cosh \pi \rho_{i} \hat{\sigma}_{i}},
$$

and setting $\bar{N}_{\mathrm{f}}=N_{\mathrm{f}}$, the matter contribution simplifies, leaving the matrix model to be

$$
Z_{N_{\mathrm{f}}}^{\mathrm{U}(N)}=\frac{1}{(2 \pi)^{N} N !} \int d^{N} \mu \frac{\prod_{i<j} 4 \sinh ^{2}\left(\frac{1}{2}\left(\mu_{i}-\mu_{j}\right)\right) e^{-\frac{1}{2 g} \sum_{i} \mu_{i}^{2}+\mathrm{i} \eta \sum_{i} \mu_{i}}}{\prod_{i}\left(2 \cosh \left(\frac{1}{2}\left(\mu_{i}+m\right)\right)\right)^{N_{\mathrm{f}}}},
$$

where $g=\frac{2 \pi \mathrm{i}}{k}$ with $k \in \mathbb{Z}$ the Chern-Simons level and $\mu_{i} / 2 \pi$ represent the eigenvalues of the scalar field $\sigma$ belonging to the three dimensional vector multiplet. In (1.3) the radius $\mathrm{R}$ of the three-sphere has been set to one. It can be restored by rescaling $m \rightarrow m \mathrm{R}, \mu_{i} \rightarrow \mu_{i} \mathrm{R}$. The partition function is periodic in imaginary shifts of the mass, $Z(m+\mathrm{i} 2 \pi n)=Z(m)$, for integer $n$. The addition of a Fayet-Iliopoulos term (FI) in the Lagrangian adds a linear term in the potential of the matrix model $[6,7,9]$. Thus $\eta$ is a real parameter denoting the FI parameter. Notice that the variables in (1.3) are rescaled with a $2 \pi$ factor with regard to those in $[6,7,9]$ and with regard to the ones in (1.1). That is, $\mu_{i}=2 \pi \sigma_{i}$.

We shall focus in this work specifically on the model (1.3) but also consider a variant of the same model, with matter content a pair of fundamental and a pair of anti-fundamental chiral multiplets ( $N_{\mathrm{f}}$ hypermultiplets of mass $m$ and $N_{\mathrm{f}}$ hypermultiplets of mass $-m$ ). The corresponding matrix model is

$$
\widetilde{Z}_{N_{\mathrm{f}}}^{\mathrm{U}(N)}=\frac{1}{(2 \pi)^{N} N !} \int d^{N} \mu \frac{\prod_{i<j} 4 \sinh ^{2}\left(\frac{1}{2}\left(\mu_{i}-\mu_{j}\right)\right) e^{-\frac{1}{2 g} \sum_{i} \mu_{i}^{2}}}{\prod_{i}\left(4 \cosh \left(\frac{1}{2}\left(\mu_{i}+m\right)\right) \cosh \left(\frac{1}{2}\left(\mu_{i}-m\right)\right)\right)^{N_{\mathrm{f}}}},
$$

which was previously studied for large $N$ in [10] and for finite $N$ and $N_{\mathrm{f}}=1$ in [11]. In this paper we consider both models, as spelled out in detail in the next section.

In [11], the approach is to express the matrix model (1.4) for $N_{\mathrm{f}}=1$, as a Hankel determinant whose entries are (combinations of) Mordell integrals [12]

$$
I(l, m)=\int_{-\infty}^{\infty} d \mu \frac{e^{(l+1) \mu+m}}{1+e^{\mu+m}} e^{-\mu^{2} / 2 g},
$$

\footnotetext{
${ }^{1}$ Notice that we have changed the sign of the Chern-Simons level with respect to that in [9] in order to make contact with our conventions.
} 
where $l \in \mathbb{C}^{2}{ }^{2}$ This integral, $I(l, m)$, was computed by Mordell [12] for general parameters. In general, it is given in terms of infinite sums of the theta-function type. However, in specific cases it assumes the form of a Gauss's finite sum [11, 12]. These specific cases precisely contain the one which is physically relevant: $g=2 \pi \mathrm{i} / k$ with $k \in \mathbb{Z}$. Exactly the same method can be applied to (1.3) and, as a matter of fact, it is simpler in that case since the identification with the Mordell integral is more direct, as we shall see below.

The main difference between [11], where analytical results for the case $N_{\mathrm{f}}=1$ were given using Mordell integrals, and this work, can be succinctly summarized by substituting (1.5) for

$$
J(l, m)=\int_{-\infty}^{\infty} d \mu \frac{e^{(l+1) \mu+m}}{\left(1+e^{\mu+m}\right)^{N_{\mathrm{f}}}} e^{-\mu^{2} / 2 g},
$$

as the main building block in all computations. This allows us to do the same computations as in [11], but also for higher flavour cases $N_{\mathrm{f}} \geq 1$ and for both all the above-mentioned properties for (1.6), following from those of (1.5). We will achieve this by using (1.5) and systematically differentiating under the integral sign, establishing also a recurrence relationship between the different derivatives. At this stage, it is worth mentioning that Mordell integrals have been applied before in problems of theoretical physics, in particular, in the study of superconformal algebras $[13,14]$. In number theory, they have become especially prominent in the last decade, after [15], due to their intimate relationship with Mock theta functions and also due to their modular properties. For example, in [16] we find an analysis of roughly the same generalization of the integral, namely (1.6), but not in the physical setting required to study (1.3). ${ }^{3}$

The main use of the formulas derived, apart from the computation of the partition functions (1.3) and (1.4), is as a tool to further analyze a Seiberg-like duality in a $3 \mathrm{~d}$ theory $[17,18]$. The two main types of Seiberg-like dualities in 3d are:

1. Aharony duality [18] for three dimensions, which holds for Chern-Simons level $k=0$ and is characterized by an unusual coupling between electric and magnetic monopoles.

2. Giveon-Kutasov duality [17], which applies to theories with any Chern-Simons level and resembles a $4 \mathrm{~d}$ Seiberg duality for theories with fundamental matter or an adjoint field. This allows for compact expressions for the observables of the Chern-Simonsmatter theory as was shown in [11] and is discussed here as well.

These two dualities can be related by starting with the Aharony duality and adding masses and generating Chern-Simons terms, leading to the Giveon-Kutasov duality. The reverse renormalization group flow from Giveon-Kutasov duality in the UV to Aharony duality in the IR has been studied in [19]. We shall focus here on the Giveon-Kutasov duality, which implies for the partition function [20]

$$
Z_{N_{\mathrm{f}}, k}^{\mathrm{U}\left(N_{\mathrm{c}}\right)}(\eta)=e^{\operatorname{sgn}(k) \pi \mathrm{i}\left(c_{|k|, N_{\mathrm{f}}}-\eta^{2}\right)} Z_{N_{\mathrm{f}},-k}^{\mathrm{U}\left(|k|+N_{\mathrm{f}}-N_{\mathrm{c}}\right)}(-\eta),
$$

\footnotetext{
${ }^{2}$ That will be the case if there is a FI parameter, see below. In simpler settings, such as in [11] and also below, it may only be an integer.

${ }^{3}$ The analysis in [16] focusses on real values of the parameters in the exponential in the context of a heat-kernel expansion, which is not the physical setting needed in the analysis of our matrix models.
} 
where the l.h.s. denotes the partition function of a theory with $N_{\mathrm{c}}$ colors, $N_{\mathrm{f}}$ hypermultiplets, Chern-Simons level $k$, and a Fayet-Iliopoulos term $\eta$. The term $c_{|k|, N_{\mathrm{f}}}$ is a phase, which is a quadratic polynomial in $k$. The principal result is an explicit expression, characterized by modular arithmetic $(\bmod 4)$ behavior, for the phase factor in (1.7). Previous, conjectured results, for this phase factor can be found in [20-22].

The paper is organized as follows. In the next section we use the determinant formulation of [11], applying it to (1.3) while also extending it to the case $N_{\mathrm{f}}>1$. This extension is based on explicit finite Gauss sums expressions for the generalized Mordell integral (1.6) that we obtain. In section 3, we use such analytical results, together with their practical implementation in Mathematica, to obtain exact analytical expressions for both (1.3) and (1.4) for a number of values of $\left(N, N_{\mathrm{f}}, k\right)$ which leads also to discuss some physical interpretations in terms of symmetry protected phases [23, 24] and mathematical features like a complex conjugacy property under the transformation $k \rightarrow-k$.

Finally, in the last section, we use the formalism developed to perform an exhaustive test of Giveon-Kutasov duality in the $\mathcal{N}=3$ setting, by explicit and systematic computation of the matrix model (1.3) on both sides of (1.7). We propose an explicit expression of the phase factor in (1.7), which is different from previous expressions in the literature [2022 ] and that we have tested to hold for a large range of the parameter space, going up to 12 flavours.

\section{Parametric derivatives of Mordell integrals for the arbitrary $N_{\mathrm{f}}$ setting}

Let us develop the method based on generalized Mordell integrals in order to compute (1.3) for higher flavour $N_{\mathrm{f}}>1$, therefore extending the methodology and results in [11]. It is enough to directly consider the derivatives of a single Mordell integral. More precisely, by making the change of variables

$$
z_{i}=c e^{\mu_{i}}, \quad c=e^{g\left(N-\frac{N_{\mathrm{f}}}{2}\right)},
$$

we may write (1.3) in the form

$$
Z_{N_{\mathrm{f}}}^{\mathrm{U}(N)}=\frac{e^{-\frac{g N}{2}\left(N-\frac{N_{\mathrm{f}}}{2}\right)\left(N+\frac{N_{\mathrm{f}}}{2}+2 \mathrm{i} \eta\right)}}{(2 \pi)^{N} N !} \int_{[0, \infty)^{N}} d^{N} z \prod_{i<j}\left(z_{i}-z_{j}\right)^{2} \frac{e^{-\frac{1}{2 g} \sum_{i}\left(\ln z_{i}\right)^{2}+\mathrm{i} \eta \sum_{i} \ln z_{i}}}{\prod_{i}\left(1+\frac{z_{i} e^{m}}{c}\right)^{N_{\mathrm{f}}}}
$$

Its determinantal formulation becomes

$$
Z_{N_{\mathrm{f}}}^{\mathrm{U}(N)}=\frac{e^{\frac{N N_{\mathrm{f}} m}{2}} c^{-\frac{N}{2}\left(N+\frac{N_{\mathrm{f}}}{2}+2 i \eta\right)}}{(2 \pi)^{N}} \operatorname{det}\left(\left(g_{i}, g_{j}\right)\right)_{i, j=0}^{N-1}
$$

where the matrix elements are given by

$$
\left(g_{i}, g_{j}\right) \equiv c^{i+j+1+\mathrm{i} \eta} e^{-\frac{1}{2 g}(\ln c)^{2}} \int_{-\infty}^{\infty} d \mu \frac{\left.e^{\mu\left(i+j+1-N+\frac{N_{\mathrm{f}}}{2}+\mathrm{i} \eta\right.}\right)}{\left(1+e^{\mu+m}\right)^{N_{\mathrm{f}}}} e^{-\frac{1}{2 g} \mu^{2}}
$$


The matrix elements may also be written in terms of a Mordell integral as follows

$$
\left(g_{i}, g_{j}\right)=c^{i+j+1+\mathrm{i} \eta} e^{-\frac{1}{2 g}(\ln c)^{2}} \frac{(-1)^{N_{\mathrm{f}}-1} e^{-m}}{\left(N_{\mathrm{f}}-1\right) !} \frac{d}{d m}\left(e^{-m} \frac{d}{d m}\left(e^{-m} \frac{d}{d m}\left(e^{-m} \frac{d}{d m} \cdots \frac{d}{d m}\left(e^{-m} I(l, m)\right)\right)\right)\right),
$$

where the derivative has to be applied $N_{\mathrm{f}}-1$ times and $l=i+j+1-N-\frac{N_{\mathrm{f}}}{2}+\mathrm{i} \eta$. Alternatively, one may exploit the relation

$$
\int_{-\infty}^{\infty} d \mu \frac{e^{\left(l+N_{\mathrm{f}}\right) \mu} e^{-\mu^{2} / 2 g}}{\left(1+e^{\mu+m}\right)^{N_{\mathrm{f}}}}=\frac{(-1)^{N_{\mathrm{f}}} e^{-m N_{\mathrm{f}}}}{\left(N_{\mathrm{f}}-1\right) !} \sum_{n=0}^{N_{\mathrm{f}}-1} C_{N_{\mathrm{f}}-1, n} I^{(n)}(l, m)
$$

and hence express (2.3) as follows

$$
\left(g_{i}, g_{j}\right)=c^{i+j+1+\mathrm{i} \eta} e^{-\frac{1}{2 g}(\ln c)^{2}} \frac{(-1)^{N_{\mathrm{f}}}}{\left(N_{\mathrm{f}}-1\right) !} e^{-m N_{\mathrm{f}}} \sum_{n=0}^{N_{\mathrm{f}}-1} C_{N_{\mathrm{f}}-1, n} I^{(n)}(l, m),
$$

where $I^{(n)}$ stands for the $n$-th derivative of $I(l, m)$ with respect to $m$ and the coefficients $C_{p, q}$ satisfy

$$
C_{p, q}= \begin{cases}-p C_{p-1, q}+C_{p-1, q-1}, & p>q>0, \\ (-1)^{p+1} p !, & p>q=0, \\ -1, & p=q .\end{cases}
$$

As it was shown in [11], (1.5) is proportional to the Mordell integral

$$
I(l, m)=2 \pi e^{-m l+\mathrm{i} k m^{2} /(4 \pi)} \int_{-\infty}^{\infty} d t \frac{e^{\mathrm{i} \pi k t^{2}+2 \pi t(l+1)-\mathrm{i} t k m}}{e^{2 \pi t}+1}
$$

and has the following explicit expression [11]

$$
I(l, m)=2 \pi\left\{\begin{array}{ll}
e^{-\mathrm{i} \pi\left(l+\frac{k}{4}\right)} e^{-m\left(l+\frac{k}{2}\right)+\frac{\mathrm{i} k m^{2}}{4 \pi}} G_{+}\left(k, 1,-l-1+\mathrm{i} \frac{k m}{2 \pi}-\frac{k}{2}\right), & k>0, \\
e^{\mathrm{i} \pi\left(l-\frac{k}{4}\right)} e^{-m\left(l-\frac{k}{2}\right)+\frac{\mathrm{i} k m^{2}}{4 \pi}} G_{-}\left(-k, 1,-l-1+\mathrm{i} \frac{k m}{2 \pi}+\frac{k}{2}\right), & k<0,
\end{array},\right.
$$

with

$$
\begin{gathered}
G_{+}\left(k, 1,-l-1+\mathrm{i} \frac{k m}{2 \pi}-\frac{k}{2}\right)=\frac{1}{e^{-2 \pi \mathrm{i} l-k m}-1}\left(-\sqrt{\frac{\mathrm{i}}{k}} \sum_{r=1}^{k} e^{\frac{\mathrm{i} \pi}{k}\left(r-l-1-\frac{k}{2}+\mathrm{i} \frac{k m}{2 \pi}\right)^{2}}+\mathrm{i}\right), \\
G_{-}\left(-k, 1,-l-1+\mathrm{i} \frac{k m}{2 \pi}+\frac{k}{2}\right)=\frac{1}{1-e^{2 \pi \mathrm{i} l+k m}}\left(\sqrt{\frac{\mathrm{i}}{k}} e^{2 \pi \mathrm{i} l+k m} \sum_{r=1}^{-k} e^{\mathrm{i} \frac{\pi}{k}\left(r+l-\frac{k}{2}-\mathrm{i} \frac{k m}{2 \pi}\right)^{2}}+\mathrm{i}\right) .
\end{gathered}
$$

Notice that, if $l \in \mathbb{Z}$, the denominator in, say, $G_{+}(a, b, x)$ could vanish in principle. However, a Gauss's sum identity [11, eq. (2.28)] prevents the partition function to diverge. We will see that for $N_{\mathrm{f}}$ odd we get $l \in \mathbb{Z} / 2$ (if we set the FI parameter to zero); in that case the identity does not apply, but the factor $e^{-2 \pi \mathrm{il}} \neq 1$ implies that the denominator does 
not vanish. Notice that the prefactor of $G_{+}$in [11] is slightly different. This is due to the fact that, there, we discussed the model with $2 N_{\mathrm{f}}$ hypermultiplets, which we study later in section 2.1 for $N_{\mathrm{f}}>1$.

The first derivative of the Mordell integral (2.7) takes the form

$$
I^{\prime}(l, m)=\left\{\begin{array}{cc}
e^{-\mathrm{i} \pi\left(l+\frac{k}{4}\right)} e^{-m\left(l+\frac{k}{2}\right)+\frac{\mathrm{i} k m^{2}}{4 \pi}\left((\mathrm{i} k m-(k+2 l) \pi) G_{+}\left(k, 1,-l-1+\mathrm{i} \frac{k m}{2 \pi}-\frac{k}{2}\right)\right.} & \\
\left.+2 \pi G_{+}^{\prime}\left(k, 1,-l-1+\mathrm{i} \frac{k m}{2 \pi}-\frac{k}{2}\right)\right), & k>0, \\
e^{\mathrm{i} \pi\left(l-\frac{k}{4}\right)} e^{-m\left(l-\frac{k}{2}\right)+\frac{\mathrm{i} k m^{2}}{4 \pi}}\left((\mathrm{i} k m+(k-2 l) \pi) G_{-}\left(-k, 1,-l-1+\mathrm{i} \frac{k m}{2 \pi}+\frac{k}{2}\right)\right. & \\
\left.+2 \pi G_{-}^{\prime}\left(-k, 1,-l-1+\mathrm{i} \frac{k m}{2 \pi}+\frac{k}{2}\right)\right), & k<0,
\end{array}\right.
$$

where we also have the Gauss sums

$$
\begin{aligned}
G_{+}^{\prime}\left(k, 1,-l-1+\mathrm{i} \frac{k m}{2 \pi}-\frac{k}{2}\right)= & \frac{e^{k m+2 i l \pi}}{\left(-1+e^{k m+2 \mathrm{i} l \pi}\right)^{2}}\left(\sqrt { \frac { \mathrm { i } } { k } } \left(-k \sum_{r=1}^{k} e^{\frac{\mathrm{i} \pi}{k}\left(-1-\frac{k}{2}-l+\frac{\mathrm{i} k m}{2 \pi}+r\right)^{2}}\right.\right. \\
& \left.\left.+\left(e^{k m+2 \mathrm{i} l \pi}-1\right) \sum_{r=1}^{k} e^{\frac{\mathrm{i} \pi}{k}\left(-1-\frac{k}{2}-l+\frac{\mathrm{i} k m}{2 \pi}+r\right)^{2}}\left(1+\frac{k}{2}+l-\frac{\mathrm{i} k m}{2 \pi}-r\right)\right)+\mathrm{i} k\right), \\
G_{-}^{\prime}\left(-k, 1,-l-1+\mathrm{i} \frac{k m}{2 \pi}+\frac{k}{2}\right)= & \frac{e^{k m+2 \mathrm{i} l \pi}}{\left(-1+e^{k m+2 \mathrm{i} l \pi}\right)^{2}}\left(\sqrt { \frac { \mathrm { i } } { k } } \left(k \sum_{r=1}^{-k} e^{\frac{\mathrm{i} \pi}{k}\left(-\frac{k}{2}+l-\frac{\mathrm{i} k m}{2 \pi}+r\right)^{2}}\right.\right. \\
& \left.\left.-\left(-1+e^{k m+2 \mathrm{i} l \pi}\right) \sum_{r=1}^{-k} e^{\frac{\mathrm{i} \pi}{k}\left(-\frac{k}{2}+l-\frac{\mathrm{i} k m}{2 \pi}+r\right)^{2}}\left(-\frac{k}{2}+l-\frac{\mathrm{i} k m}{2 \pi}+r\right)\right)+\mathrm{i} k\right) .
\end{aligned}
$$

\subsection{The theory with $2 N_{\mathrm{f}}$ hypermultiplets}

We now consider the case of $N_{\mathrm{f}}$ hypermultiplets with vector mass $m$ and $N_{\mathrm{f}}$ hypermultiplets with vector mass $-m$. This theory was analyzed in detail in [11] for $N_{\mathrm{f}}=1$ and previously in [10]. One of its distinctive features is the existence of third order phase transitions in a certain double scaling limit which involves a decompactification limit, in which the radius of $\mathbb{S}^{3}$ is taken to infinity $[10,11]$. The partition function, expressed as a matrix integral, is

$$
\widetilde{Z}_{N_{\mathrm{f}}}^{\mathrm{U}(N)}=\frac{1}{(2 \pi)^{N} N !} \int d^{N} \mu \frac{\prod_{i<j} 4 \sinh ^{2}\left(\frac{1}{2}\left(\mu_{i}-\mu_{j}\right)\right) e^{-\frac{1}{2 g} \sum_{i} \mu_{i}^{2}}}{\prod_{i}\left(4 \cosh \left(\frac{1}{2}\left(\mu_{i}+m\right)\right) \cosh \left(\frac{1}{2}\left(\mu_{i}-m\right)\right)\right)^{N_{\mathrm{f}}}},
$$

where for simplicity (and to compare with [11]) we have not included the FI term. The simple change of variables is $[10,11]$

$$
z_{i}=c e^{\mu_{i}}, \quad c=e^{g\left(N-N_{\mathrm{f}}\right)},
$$

which recasts the integral in the form

$$
\widetilde{Z}_{N_{\mathrm{f}}}^{\mathrm{U}(N)}=\frac{e^{-\frac{g N}{2}\left(N^{2}-N_{\mathrm{f}}^{2}\right)} e^{2 g N N_{\mathrm{f}}}}{(2 \pi)^{N} N !} \int_{[0, \infty)^{N}} d^{N} z \prod_{i<j}\left(z_{i}-z_{j}\right)^{2} \frac{e^{-\frac{1}{2 g} \sum_{i}\left(\ln z_{i}\right)^{2}}}{\prod_{i}\left(c e^{-m}+z_{i}\right)^{N_{\mathrm{f}}}\left(c e^{m}+z_{i}\right)^{N_{\mathrm{f}}}} .
$$


As in [11], the partition function can be written as

$$
\widetilde{Z}_{N_{\mathrm{f}}}^{\mathrm{U}(N)}=N ! e^{-\frac{g N}{2}\left(N^{2}-N_{\mathrm{f}}^{2}\right)} \operatorname{det}\left(\left(f_{i}, f_{j}\right)\right)_{i, j=0}^{N-1}
$$

where the functions $f_{i}$ have the form

$$
\left(f_{i}, f_{j}\right)=c^{i+j+1} e^{-(\ln c)^{2} / 2 g} \int_{-\infty}^{+\infty} d \mu \frac{e^{i+j+1+N_{\mathrm{f}}-N}}{\left(1+e^{\mu+m}\right)^{N_{\mathrm{f}}}\left(1+e^{\mu-m}\right)^{N_{\mathrm{f}}}} e^{-\mu^{2} / 2 g} .
$$

The objective is to compute (2.15) for generic $N_{\mathrm{f}}$ and then the partition function follows from (2.14). First we expand the denominator in (2.15) using the identity

$$
\begin{aligned}
\frac{1}{(1+a x)^{n}(1+b x)^{n}}= & \frac{1}{(a-b)^{n}} \sum_{s=0}^{n-1}\left(\begin{array}{c}
n+s-1 \\
s
\end{array}\right)\left(\frac{a b}{a-b}\right)^{s}\left((-1)^{s}\left(\frac{a}{1+a x}\right)^{n-s}\right. \\
& \left.+(-1)^{n}\left(\frac{b}{1+b x}\right)^{n-s}\right) .
\end{aligned}
$$

This is a generalization for $n>1$ of the identity used in [11]. Using the summation form of (2.16) and setting $\ell=i+j+1-N$, we are able to write (2.15) as

$$
\begin{aligned}
\left(f_{i}, f_{j}\right)= & \frac{c^{i+j+1} e^{-(\ln c)^{2} / 2 g}}{2^{N_{\mathrm{f}}}(\sinh m)^{N_{\mathrm{f}}}} \sum_{s=0}^{N_{\mathrm{f}}-1}\left(\begin{array}{c}
N_{\mathrm{f}}+s-1 \\
s
\end{array}\right) \frac{1}{(2 \sinh m)^{s}} \\
& \times\left((-1)^{s} e^{m\left(N_{\mathrm{f}}-s-1\right)} \int_{-\infty}^{\infty} d \mu \frac{e^{\mu\left(\ell+N_{\mathrm{f}}\right)+m} e^{-\mu^{2} / 2 g}}{\left(1+e^{\mu+m}\right)^{N_{\mathrm{f}}-s}}\right. \\
& \left.+(-1)^{N_{\mathrm{f}}} e^{-m\left(N_{\mathrm{f}}-s-1\right)} \int_{-\infty}^{\infty} d \mu \frac{e^{\mu\left(\ell+N_{\mathrm{f}}\right)-m} e^{-\mu^{2} / 2 g}}{\left(1+e^{\mu-m}\right)^{N_{\mathrm{f}}-s}}\right) .
\end{aligned}
$$

The integrals in (2.17) can be expressed in terms of a Mordell integral (1.5). Specifically, we note that

$$
\begin{aligned}
& \frac{d}{d m}\left(e^{-m} \frac{d}{d m}\left(e^{-m} \frac{d}{d m} \cdots \frac{d}{d m}\left(e^{-m} I(\ell+s, m)\right)\right)\right) \\
& \quad=(-1)^{N_{\mathrm{f}}-s-1}\left(N_{\mathrm{f}}-s-1\right) ! \int_{-\infty}^{\infty} \frac{e^{\mu\left(\ell+N_{\mathrm{f}}\right)+m} e^{-\mu^{2} / 2 g}}{\left(1+e^{\mu+m}\right)^{N_{\mathrm{f}}-s}} \\
& \frac{d}{d m}\left(e^{m} \frac{d}{d m}\left(e^{m} \frac{d}{d m} \cdots \frac{d}{d m}\left(e^{m} I(\ell+s,-m)\right)\right)\right) \\
& \quad=\left(N_{\mathrm{f}}-s-1\right) ! \int_{-\infty}^{\infty} \frac{e^{\mu\left(\ell+N_{\mathrm{f}}\right)-m} e^{-\mu^{2} / 2 g}}{\left(1+e^{\mu-m}\right)^{N_{\mathrm{f}}-s}}
\end{aligned}
$$

where the derivative on the left hand side of the above expressions is applied $N_{\mathrm{f}}-s-1$ times. These expressions help us to alternatively express (2.17) as a sum of derivatives 
of (1.5) with respect to $m$. Particularly,

$$
\begin{aligned}
& \int_{-\infty}^{\infty} d \mu \frac{e^{\left(\ell+N_{\mathrm{f}}\right) \mu} e^{-\mu^{2} / 2 g}}{\left(1+e^{\mu+m}\right)^{N_{\mathrm{f}}-s}}=\frac{(-1)^{N_{\mathrm{f}}-s} e^{-\left(N_{\mathrm{f}}-s\right) m}}{\left(N_{\mathrm{f}}-s-1\right) !} \sum_{n=0}^{N_{\mathrm{f}}-s-1} C_{N_{\mathrm{f}}-s-1, n}^{(+)} I^{(n)}(\ell+s, m), \\
& \int_{-\infty}^{\infty} d \mu \frac{e^{\left(\ell+N_{\mathrm{f}}\right) \mu} e^{-\mu^{2} / 2 g}}{\left(1+e^{\mu-m}\right)^{N_{\mathrm{f}}-s}}=\frac{e^{\left(N_{\mathrm{f}}-s\right) m}}{\left(N_{\mathrm{f}}-s-1\right) !} \sum_{n=0}^{N_{\mathrm{f}}-s-1} C_{N_{\mathrm{f}}-s-1, n}^{(-)} I^{(n)}(\ell+s,-m),
\end{aligned}
$$

where $I^{(n)}(\ell, m) \equiv \frac{d^{n} I(\ell, m)}{d m^{n}}$. The constant factors $C_{p, q}^{( \pm)}$are given by the recursion relations

$$
\begin{aligned}
& C_{p, q}^{(+)}= \begin{cases}-p C_{p-1, q}^{(+)}+C_{p-1, q-1}^{(+)}, & p>q>0, \\
(-1)^{p+1} p !, & p>q=0, \\
-1, & p=q .\end{cases} \\
& C_{p, q}^{(-)}= \begin{cases}p C_{p-1, q}^{(-)}+C_{p-1, q-1}^{(-)}, & p>q>0, \\
p !, & p>q=0, \\
1, & p=q,\end{cases}
\end{aligned}
$$

respectively. To summarize

$$
\begin{aligned}
\left(f_{i}, f_{j}\right)= & \frac{c^{i+j+1} e^{-(\ln c)^{2} / 2 g}(-1)^{N_{\mathrm{f}}}}{2^{N_{\mathrm{f}}(\sinh m)^{N_{\mathrm{f}}}}} \sum_{s=0}^{N_{\mathrm{f}}-1}\left(\begin{array}{c}
N_{\mathrm{f}}+s-1 \\
s
\end{array}\right) \frac{1}{(2 \sinh m)^{s}\left(N_{\mathrm{f}}-s-1\right) !} \\
& \times\left(\sum_{n=0}^{N_{\mathrm{f}}-s-1} C_{N_{\mathrm{f}}-s-1, n}^{(+)} I^{(n)}(\ell+s, m)+\sum_{n=0}^{N_{\mathrm{f}}-s-1} C_{N_{\mathrm{f}}-s-1, n}^{(-)} I^{(n)}(\ell+s,-m)\right)
\end{aligned}
$$

where the derivatives of $I(\ell+s, m)$ are estimated from (2.7) as before.

\section{Analytical expressions for the partition functions and some interpre- tations}

We put the formalism we have just developed into use and compute, as in [11], specific instances of the partition function of both models (1.3) and (1.4) for $N_{\mathrm{f}} \geq 1$. In general, we restrict ourselves with presenting the cases comprising $\mathrm{U}(1), \mathrm{U}(2)$ and $\mathrm{U}(3)$, with $N_{\mathrm{f}}=$ $1,2,3, \eta=0$ and also $|k|=1,2,3$.

\subsection{Abelian partition functions}

The Abelian partition function is given directly by Mordell's integral. In the case $N_{\mathrm{f}}=2$ and $k$ arbitrary, we can use the first derivative of the Mordell integral to obtain an explicit 
expression

$$
\begin{aligned}
Z_{k, N_{\mathrm{f}}=2}^{\mathrm{U}(1)}(\eta) & =\frac{e^{-m}}{2 \pi}\left(I(\ell=-1+\mathrm{i} \eta, m)-I^{\prime}(\ell=-1+\mathrm{i} \eta, m)\right) \\
& =\left\{\begin{array}{cc}
-e^{-\mathrm{i} \pi\left(\mathrm{i} \eta+\frac{k}{4}\right)} e^{-m\left(\mathrm{i} \eta+\frac{k}{2}\right)+\frac{\mathrm{i} k m^{2}}{4 \pi}}\left(\left(\mathrm{i} \eta-\frac{\mathrm{i} k m}{2 \pi}+\frac{k}{2}\right) G_{+}\left(k, 1,-\mathrm{i} \eta+\mathrm{i} \frac{k m}{2 \pi}-\frac{k}{2}\right)\right. & k>0, \\
\left.-G_{+}^{\prime}\left(k, 1,-\mathrm{i} \eta+\mathrm{i} \frac{k m}{2 \pi}-\frac{k}{2}\right)\right), & k<0, \\
-e^{\mathrm{i} \pi\left(\mathrm{i} \eta-\frac{k}{4}\right)} e^{-m\left(\mathrm{i} \eta-\frac{k}{2}\right)+\frac{\mathrm{i} k m^{2}}{4 \pi}}\left(\left(\mathrm{i} \eta-\frac{\mathrm{i} k m}{2 \pi}-\frac{k}{2}\right) G_{-}\left(-k, 1,-\mathrm{i} \eta+\mathrm{i} \frac{k m}{2 \pi}+\frac{k}{2}\right)\right. & k \\
\left.-G_{-}^{\prime}\left(-k, 1,-\mathrm{i} \eta+\mathrm{i} \frac{k m}{2 \pi}+\frac{k}{2}\right)\right), &
\end{array}\right.
\end{aligned}
$$

where the Gauss sums are again (2.8) and (2.10). As pointed out in [11], the formulae contain perturbative as well as non-perturbative terms. The perturbative terms arise from the weak-coupling expansion of factors $e^{\frac{\mathrm{i} \pi}{k}(r-1)^{2}}=e^{\frac{g}{2}(r-1)^{2}}$, whereas non-perturbative terms are factors $e^{\frac{\mathrm{i} k(m-\mathrm{i} \pi)^{2}}{4 \pi}}=e^{-\frac{(m-\mathrm{i} \pi)^{2}}{2 g}}$ and $e^{k m}=e^{\frac{2 \pi \mathrm{i} m}{g}}$. For specific values of $k$, and flavour $N_{\mathrm{f}}=1$, we obtain

$$
\begin{aligned}
Z_{k=1, N_{\mathrm{f}}=1}^{\mathrm{U}(1)} & =\frac{e^{m / 2} e^{\mathrm{i} \frac{\pi}{2}}\left(1-e^{\mathrm{i} \frac{\pi}{4}} e^{\frac{\mathrm{i} m^{2}}{4 \pi}}\right)}{e^{m}+1}, \\
Z_{k=-1, N_{\mathrm{f}}=1}^{\mathrm{U}(1)} & =\frac{e^{m / 2}\left(-e^{\mathrm{i} \frac{\pi}{2}}+e^{\mathrm{i} \frac{\pi}{4}} e^{-\frac{\mathrm{i} m^{2}}{4 \pi}}\right)}{e^{m}+1}, \\
Z_{k=2, N_{\mathrm{f}}=1}^{\mathrm{U}(1)} & =\frac{e^{\frac{\pi \mathrm{i}}{2}} e^{m / 2}\left(-\sqrt{2} e^{\frac{m(\mathrm{i} m+\pi)}{2 \pi}}+e^{-\frac{\pi \mathrm{i}}{8}}\left(1+e^{m}\right)\right)}{\sqrt{2}\left(1+e^{2 m}\right)}, \\
Z_{k=-2, N_{\mathrm{f}}=1}^{\mathrm{U}(1)} & =-\frac{\sqrt{2} e^{m / 2} e^{\frac{5 \mathrm{i} \pi}{8}}\left(1+e^{m}\right)-2 \mathrm{i} e^{m} e^{-\frac{\mathrm{i} m^{2}}{2 \pi}}}{2\left(e^{2 m}+1\right)}, \\
Z_{k=3, N_{\mathrm{f}}=1}^{\mathrm{U}(1)} & =\frac{e^{3 m / 2}\left(1 / \sqrt{3}-e^{\mathrm{i} \pi / 4} e^{\frac{3 i m^{2}}{4 \pi}}+\left(e^{\mathrm{i} \pi / 2}+1 / \sqrt{3}\right) \cosh m\right)}{\left(e^{3 m}+1\right)} .
\end{aligned}
$$

Notice that there seem to be apparent poles at $m=\mathrm{i} \pi / k$ and, even though masses are real, one can still look for poles or zeros of the partition function on the complex plane, see $[25,26]$ for example. As we shall see below with more cases, these supposed poles seem to appear for $m=2 \mathrm{i} \pi / k$ for $N_{\mathrm{f}}$ even and $m=\mathrm{i} \pi / k$ for odd $N_{\mathrm{f}}$. That is actually not the case, and the partition function is smooth at these points, as expected. We explicitly compute and check that the derivatives are smooth functions of the mass too. 
As a matter of fact, it is known that the Mordell integral (1.5) is an holomorphic function ${ }^{4}$ in $l$. Since the Abelian partition functions are directly Mordell integrals, then they are holomorphic in that parameter, which comprises both the mass ${ }^{5}$ and the FI parameter. The same implication holds for the non-Abelian case, since it is a determinant of holomorphic functions.

In the $k=1$ case, the duality is between Abelian theories and we indeed check that

$$
\frac{Z_{k=1, N_{\mathrm{f}}=1}^{\mathrm{U}(1)}}{Z_{k=-1, N_{\mathrm{f}}=1}^{\mathrm{U}(1)}}=e^{\frac{\mathrm{i} \pi}{4}+\frac{\mathrm{i} m^{2}}{4 \pi}} .
$$

Likewise, the cases $k=2$ and $k=3$ above, will be related with duals below, with gauge group $\mathrm{U}(2)$ and $\mathrm{U}(3)$ respectively. For $N_{\mathrm{f}}=2$ we have

$$
\begin{aligned}
Z_{k=1, N_{\mathrm{f}}=2}^{\mathrm{U}(1)} & =\frac{e^{\mathrm{i} \frac{\pi}{4}}\left(-\pi+e^{\frac{\mathrm{i} m^{2}}{4 \pi}}(\pi \cosh (m / 2)-\mathrm{i} m \sinh (m / 2))\right)}{2 \pi(\cosh m-1)}, \\
Z_{k=-1, N_{\mathrm{f}}=2}^{\mathrm{U}(1)} & =\frac{-e^{m / 2} e^{-\frac{\mathrm{i} m^{2}}{4 \pi}}\left(2 \pi e^{\frac{\mathrm{i}(m-\mathrm{i} \pi)^{2}}{4 \pi}}+e^{\mathrm{i} \pi / 4}\left(m-e^{m} m+\mathrm{i} \pi\left(1+e^{m}\right)\right)\right)}{2 \pi\left(e^{m}-1\right)^{2}}, \\
Z_{k=2, N_{\mathrm{f}}=2}^{\mathrm{U}(1)} & =\frac{e^{\frac{m}{2}\left(6+\frac{\mathrm{i} m}{\pi}\right)}(\pi-\mathrm{i} m)+e^{m+\frac{\mathrm{i} m^{2}}{2 \pi}}(\pi+\mathrm{i} m)-(1-\mathrm{i}) \pi e^{2 m}(\cosh m+\mathrm{i})}{\pi\left(e^{2 m}-1\right)^{2}}, \\
Z_{k=-2, N_{\mathrm{f}}=2}^{\mathrm{U}(1)} & =\frac{-\sqrt{2} e^{\frac{\pi \mathrm{i}}{4}} \pi e^{2 m}(\cosh m-\mathrm{i})+2 e^{\frac{m}{2}\left(4-\frac{\mathrm{i} m}{\pi}\right)}(\pi \cosh m+\mathrm{i} m \sinh m)}{\pi\left(e^{2 m}-1\right)^{2}} .
\end{aligned}
$$

It is not manifest from the form in which the partition functions are given (for example, in the last two expressions above) but the partition functions satisfy

$$
Z\left(N_{\mathrm{c}}, N_{\mathrm{f}}, k, m, \eta\right)=\bar{Z}\left(N_{\mathrm{c}}, N_{\mathrm{f}},-k, m,-\eta\right),
$$

where $\bar{Z}$ denotes the complex conjugate. In the massless case it also holds that

$$
Z\left(N_{\mathrm{c}}, N_{\mathrm{f}}, k, m=0, \eta\right)=\bar{Z}\left(N_{\mathrm{c}}, N_{\mathrm{f}},-k, m=0, \eta\right),
$$

because the partition function can be shown to be an even function in the FI parameter. We have checked these properties explicitly in all the cases analyzed, both analytically and numerically as well up to $N_{\mathrm{f}}=12$. A rigorous proof is immediate, but since it has more implications, it will be given elsewhere. As an example, let us rewrite the two examples above, showcasing their real and imaginary parts, which we respectively name $Z_{r}$ and $Z_{I}$

$$
Z_{k= \pm 2, N_{\mathrm{f}}=2}^{\mathrm{U}(1)}=Z_{r} \mp \mathrm{i} Z_{I}
$$

\footnotetext{
${ }^{4}$ We write (1.5) in the equivalent form $h(z, \tau)=\int_{\mathbb{R}} d x \exp \left(\pi \mathrm{i} \tau x^{2}-2 \pi z x\right) / \cosh \pi x$, then, with $z \in \mathbb{C}$ and $\tau \in \mathbb{H}$. It is the only holomorphic function in $z$ which satisfies [15] $h(z)+h(z+1)=\frac{2}{\sqrt{-\mathrm{i} \tau}} e^{\pi \mathrm{i}(z+1 / 2)^{2} / \tau}$ and $h(z)+e^{-2 \pi \mathrm{i} z-\pi \mathrm{i} \tau} h(z+\tau)=2 e^{-\pi \mathrm{i} z-\pi \mathrm{i} \tau / 4}$. It also satisfies an S-modular property, which can be used to obtain the Giveon-Kutasov duality in the Abelian case (3.1). Details will appear elsewhere.

${ }^{5}$ Although $l$ does not explicitly depend on $m$, we can promote the mass term into the linear part of the exponential in the numerator, by shifting the eigenvalues by the mass $m$.
} 
where

$$
\begin{aligned}
& Z_{r}=\frac{e^{2 m}}{\left(e^{2 m}-1\right)^{2}}\left(-1-\cosh m+2\left(\cos \left(\frac{m^{2}}{2 \pi}\right) \cosh m+\frac{m}{\pi} \sin \left(\frac{m^{2}}{2 \pi}\right) \sinh m\right)\right), \\
& Z_{I}=\frac{e^{2 m}}{\left(e^{2 m}-1\right)^{2}}\left(1-\cosh m-2\left(\sin \left(\frac{m^{2}}{2 \pi}\right) \cosh m-\frac{m}{\pi} \cos \left(\frac{m^{2}}{2 \pi}\right) \sinh m\right)\right) .
\end{aligned}
$$

It is well-known that, in general, the partition functions computed with the localization method are complex $[27,28]$, whereas unitarity demands the partition function to be real. See $[27,28]$ for a detailed discussion. In addition, we have seen explicitly that there is a complex conjugate property when $k \rightarrow-k$ and below we identify a family of partition functions whose complex conjugate is the partition function of the inverse field theory (see $[24,30]$ for the notion of inverse field theory).

\section{$3.2 \quad \mathrm{U}(2)$ gauge group}

We begin with the $N_{\mathrm{f}}=1$ case and consider some duals with the Abelian examples above

$$
\begin{aligned}
Z_{k=1, N_{\mathrm{f}}=1}^{\mathrm{U}(2)} & =e^{-\frac{3 \pi \mathrm{i}}{4}+\frac{\mathrm{i} m^{2}}{4 \pi}}, \\
Z_{k=2, N_{\mathrm{f}}=1}^{\mathrm{U}(2)} & =\frac{e^{-\frac{\mathrm{i} \pi}{4}}\left(2 e^{m}-\sqrt{2} e^{\frac{\mathrm{i} \pi}{8}} e^{\frac{\mathrm{i} m^{2}}{2 \pi}} e^{m / 2}\left(1+e^{m}\right)\right)}{2\left(e^{2 m}+1\right)}, \\
Z_{k=-2, N_{\mathrm{f}}=1}^{\mathrm{U}(2)} & =\frac{e^{\frac{\mathrm{i} \pi}{4}} e^{\frac{m}{2}-\frac{\mathrm{i} m^{2}}{2 \pi}}\left(2 e^{\frac{m}{2}+\frac{\mathrm{i} m^{2}}{2 \pi}}+\sqrt{2} e^{\frac{7 \mathrm{i} \pi}{8}}\left(1+e^{m}\right)\right)}{2\left(e^{2 m}+1\right)}, \\
Z_{k=-3, N_{\mathrm{f}}=1}^{\mathrm{U}(2)} & =\frac{\mathrm{i}-e^{\frac{5 \mathrm{i} \pi}{12}-\frac{3 \mathrm{i} m^{2}}{4 \pi}}}{\sqrt{3}(-1+2 \cosh m)} .
\end{aligned}
$$

Some duality cases are

$$
\frac{Z_{k=2, N_{\mathrm{f}}=1}^{\mathrm{U}(1)}}{Z_{k=-2, N_{\mathrm{f}}=1}^{\mathrm{U}(2)}}=e^{-\frac{3 \pi \mathrm{i}}{4}+\frac{\mathrm{i} m^{2}}{2 \pi}},
$$

and

$$
\frac{Z_{k=-2, N_{\mathrm{f}}=1}^{\mathrm{U}(1)}}{Z_{k=2, N_{\mathrm{f}}=1}^{\mathrm{U}(2)}}=e^{\frac{3 \pi \mathrm{i}}{4}-\frac{\mathrm{i} m^{2}}{2 \pi}},
$$

as expected, because of the complex conjugation of the partition function under the change $k \rightarrow-k$. Let us now present some $\mathrm{U}(2)$ examples with $N_{\mathrm{f}}=2$, and relate them with some of their duals above, mostly with $\mathrm{U}(1)$ and $N_{\mathrm{f}}=2$ cases

$$
\begin{aligned}
Z_{k=1, N_{\mathrm{f}}=2}^{\mathrm{U}(2)} & =\frac{e^{m / 2} e^{\frac{\mathrm{i} m^{2}}{4 \pi}}\left(m\left(1-e^{m}\right)+\mathrm{i} \pi\left(1+e^{m}-2 e^{\frac{m}{2}+\frac{\mathrm{i} m^{2}}{4 \pi}}\right)\right)}{2 \pi\left(e^{m}-1\right)^{2}}, \\
Z_{k=-1, N_{\mathrm{f}}=2}^{\mathrm{U}(2)}= & \frac{e^{m / 2} e^{-\frac{\mathrm{i} m^{2}}{2 \pi}}\left(2 \pi \mathrm{i} e^{m / 2}+e^{\frac{\mathrm{i} m^{2}}{4 \pi}}\left(m\left(1-e^{m}\right)-\mathrm{i} \pi\left(1+e^{m}\right)\right)\right)}{2 \pi\left(e^{m}-1\right)^{2}} .
\end{aligned}
$$


We can combine these particular cases to highlight a few more explicit analytical examples of Giveon-Kutasov duality. Namely,

$$
\begin{aligned}
& \frac{Z_{k=1, N_{\mathrm{f}}=2}^{\mathrm{U}(1)}}{Z_{k=-1, N_{\mathrm{f}}=2}^{\mathrm{U}(2)}}=e^{\frac{3 \pi \mathrm{i}}{4}+\frac{\mathrm{i} m^{2}}{2 \pi}}, \\
& \frac{Z_{k=-1, N_{\mathrm{f}}=2}^{\mathrm{U}(1)}}{Z_{k=1, N_{\mathrm{f}}=2}^{\mathrm{U}(2)}}=e^{-\frac{3 \pi \mathrm{i}}{4}-\frac{\mathrm{i} m^{2}}{2 \pi}} .
\end{aligned}
$$

\subsection{Supersymmetry breaking and partition functions of modulus 1}

Notice the special form of the partition function (3.3) (see also [11, eq. (2.36)]). Such partition functions arise when the dual is actually a theory with $\mathrm{U}\left(N_{\mathrm{c}}=0\right)$ and hence the partition function of the dual is just 1 . Therefore, the only term remaining is the phase factor of the Giveon-Kutasov duality (1.7).

Thus, there is a family of partition functions, satisfying $|k|-N_{\mathrm{c}}+N_{\mathrm{f}}=0$, whose value are just Giveon-Kutasov phases. This family of partition functions therefore constitute a marginal case, separating the partition functions which are identically zero, namely those which satisfy $|k|-N_{\mathrm{c}}+N_{\mathrm{f}}<0$, due to spontaneous supersymmetry breaking [29] and the regular ones (that satisfy $|k|-N_{\mathrm{c}}+N_{\mathrm{f}}>0$ ). Our determinantal method indeed directly gives null results for those cases characterized by $|k|-N_{\mathrm{c}}+N_{\mathrm{f}}<0$. In this way for example, we obtained

$$
\begin{array}{lll}
Z_{k= \pm 1, N_{\mathrm{f}}=1}^{\mathrm{U}(4)}=Z_{k= \pm 2, N_{\mathrm{f}}=1}^{\mathrm{U}(4)}=0 & \text { and } & Z_{k= \pm 3, N_{\mathrm{f}}=1}^{\mathrm{U}(4)}=e^{ \pm \frac{\mathrm{i} \pi}{12} \pm \frac{3 \mathrm{i} m^{2}}{4 \pi}} \\
Z_{k= \pm 1, N_{\mathrm{f}}=1}^{\mathrm{U}(5)}=Z_{k= \pm 2, N_{\mathrm{f}}=1}^{\mathrm{U}(5)}=Z_{k= \pm 3, N_{\mathrm{f}}=1}^{\mathrm{U}(5)}=0 & \text { and } & Z_{k= \pm 4, N_{\mathrm{f}}=1}^{\mathrm{U}(5)}=e^{ \pm \frac{7 \mathrm{i} \pi}{4} \pm \frac{\mathrm{i} m^{2}}{\pi}}
\end{array}
$$

and so on. It is noteworthy that this type of partition function, being a complex number of modulus one, is the one that emerges in the description of symmetry protected phases [23]. These partition functions $Z_{\mathbb{S}^{3}}=e^{\mathrm{i} \Phi}$ are of a topological quantum field theory which is invertible $[24,30]$, with its inverse being the theory with complex conjugate partition function $Z_{\mathbb{S}^{3}}^{-1}=e^{-\mathrm{i \Phi} \Phi}$, which in our case corresponds to $k \rightarrow-k$. Thus, for our $\mathcal{N}=2$ theory, through the Giveon-Kutasov duality, we have seen that the partition functions that exhibit such behavior come exclusively from the anomaly phase factor. This is also consistent with the idea that anomalies are invertible field theories ([30] and references therein). Further analysis of this result here from the perspective of study of the topological phases of matter seems an interesting open problem. In the next section, we actually give an analytical expression for $\Phi=\Phi\left(k, N_{\mathrm{c}}, N_{\mathrm{f}}, m, \eta\right)$.

\section{$3.4 \mathrm{U}(3)$ gauge group}

For $N_{\mathrm{f}}=1$ we have

$$
\begin{aligned}
& Z_{k=1, N_{\mathrm{f}}=1}^{\mathrm{U}(3)}=0, \\
& Z_{k=2, N_{\mathrm{f}}=1}^{\mathrm{U}(3)}=e^{-\frac{3 \pi \mathrm{i}}{4}+\frac{\mathrm{i} m^{2}}{2 \pi}},
\end{aligned}
$$




$$
\begin{aligned}
Z_{k=3, N_{\mathrm{f}}=1}^{\mathrm{U}(3)}= & \frac{e^{\frac{3 \pi \mathrm{i}}{4}} e^{m / 2}\left(6 e^{-\frac{11 \pi \mathrm{i}}{12}} e^{m}+2 \sqrt{3} e^{\frac{m}{4}\left(8+\frac{3 \mathrm{i} m}{\pi}\right)}+(3 \mathrm{i}+\sqrt{3}) e^{m+\frac{3 \mathrm{i} m^{2}}{4 \pi}}+2 \sqrt{3} e^{\frac{3 \mathrm{i} m^{2}}{4 \pi}}\right)}{6\left(e^{3 m}+1\right)}, \\
Z_{k=-3, N_{\mathrm{f}}=1}^{\mathrm{U}(3)}= & \frac{\sqrt{2} e^{-\frac{3 \pi \mathrm{i}}{4}} e^{\frac{3}{4} m\left(4-\frac{\mathrm{i} m}{\pi}\right)} \cosh (m / 2)(2 \cosh m-1)}{6\left(e^{3 m}+1\right)^{2}}\left(2 \sqrt{6} e^{-\frac{\pi \mathrm{i}}{3}}-6 \sqrt{2} e^{-\frac{\pi \mathrm{i}}{12}} e^{\frac{3 \mathrm{3i} m^{2}}{4 \pi}}\right. \\
& +4 \sqrt{6} \cosh m) .
\end{aligned}
$$

For these values of the parameters the duality now becomes

$$
\begin{gathered}
\frac{Z_{k=2, N_{\mathrm{f}}=1}^{\mathrm{U}(3)}}{Z_{k=2, N_{\mathrm{f}}=1}^{\mathrm{U}(0)}}=e^{-\frac{3 \pi \mathrm{i}}{4}+\frac{\mathrm{i} m^{2}}{2 \pi}}, \\
\frac{Z_{k=3, N_{\mathrm{f}}=1}^{\mathrm{U}(1)}}{Z_{k=-3, N_{\mathrm{f}}=1}^{\mathrm{U}(3)}}=e^{-\frac{11 \pi \mathrm{i}}{12}+\frac{3 \mathrm{i} m^{2}}{4 \pi}} .
\end{gathered}
$$

For $N_{\mathrm{f}}=2$ we have

$$
\begin{aligned}
Z_{k=1, N_{\mathrm{f}}=2}^{\mathrm{U}(3)}= & e^{\frac{3 \pi \mathrm{i}}{4}+\frac{\mathrm{i} m^{2}}{2 \pi}}, \\
Z_{k=2, N_{\mathrm{f}}=2}^{\mathrm{U}(3)}= & \frac{e^{m+\frac{\mathrm{i} m^{2}}{2 \pi}}}{\sqrt{2} \pi\left(e^{2 m}-1\right)^{2}}\left(\sqrt{2}\left(1-e^{2 m}\right) m+\sqrt{2} \pi e^{\frac{\pi \mathrm{i}}{2}}\left(1+e^{2 m}\right)+\right. \\
& \left.\pi e^{-\frac{\pi \mathrm{i}}{4}} e^{\frac{1}{2} m\left(4+\frac{\mathrm{i} m}{\pi}\right)}-2 \pi e^{\frac{\pi \mathrm{i}}{4}} e^{m+\frac{\mathrm{i} m^{2}}{2 \pi}}+\pi e^{-\frac{\pi \mathrm{i}}{4}} e^{\frac{\mathrm{i} m^{2}}{2 \pi}}\right), \\
Z_{k=3, N_{\mathrm{f}}=2}^{\mathrm{U}(3)}= & \frac{e^{\frac{3 i m^{2}}{4 \pi}}}{\sinh \left(\frac{3 m}{2}\right)}\left(\frac{\sqrt{3} m}{4 \pi} e^{\frac{7 \pi \mathrm{i}}{12}}+\frac{e^{\frac{\pi \mathrm{i}}{2}}}{2 \sqrt{2}} \operatorname{coth}\left(\frac{3 m}{2}\right)\right)+\frac{e^{7 \pi \mathrm{i} / 12}}{4 \sinh ^{2}\left(\frac{3 m}{2}\right)} \\
& +\frac{e^{\frac{3 \mathrm{i} m^{2}}{4 \pi}}}{\sqrt{3} \sinh ^{2}\left(\frac{3 m}{2}\right)}\left(e^{7 \pi \mathrm{i} / 4}\left(\frac{1}{2}+\cosh \left(\frac{m}{2}\right)\right)+\frac{\cosh (m)}{2}\right) .
\end{aligned}
$$

The duals we extract from these cases are

$$
\frac{Z_{k=-2, N_{\mathrm{f}}=2}^{\mathrm{U}(1)}}{Z_{k=2, N_{\mathrm{f}}=2}^{\mathrm{U}(3)}}=e^{\frac{3 \pi \mathrm{i}}{2}-\frac{\mathrm{i} m^{2}}{\pi}} \quad \text { and } \quad \frac{Z_{k=2, N_{\mathrm{f}}=2}^{\mathrm{U}(1)}}{Z_{k=-2, N_{\mathrm{f}}=2}^{\mathrm{U}(3)}}=e^{\frac{\pi \mathrm{i}}{2}+\frac{\mathrm{i} m^{2}}{\pi}},
$$

and we also find

$$
\frac{Z_{k=3, N_{\mathrm{f}}=2}^{\mathrm{U}(2)}}{Z_{k=-3, N_{\mathrm{f}}=2}^{\mathrm{U}(3)}}=e^{-\frac{11 \pi \mathrm{i}}{12}+\frac{3 \mathrm{im} m^{2}}{2 \pi}}
$$

\subsection{Cases with $N_{\mathrm{f}}=3$}

Instead of giving the explicit partition functions for the $N_{\mathrm{f}}=3$ case, we give the ratio of the dual partition functions directly

$$
\begin{aligned}
& \frac{Z_{k=1, N_{\mathrm{f}}=3}^{\mathrm{U}(2)}}{Z_{k=-1, N_{\mathrm{f}}=3}^{\mathrm{U}(2)}}=e^{\frac{3 \mathrm{i} \pi}{4}+\frac{3 \mathrm{i} m^{2}}{4 \pi}}, \\
& \frac{Z_{k=2, N_{\mathrm{f}}=3}^{\mathrm{U}(2)}}{Z_{k=-2, N_{\mathrm{f}}=3}^{\mathrm{U}(3)}}=e^{\frac{\mathrm{i} \pi}{4}+\frac{3 \mathrm{i} m^{2}}{2 \pi}} .
\end{aligned}
$$


Note that the duals in the former case have the same gauge group. It is immediate to check that this is always the case when, for $m, n \in \mathbb{N}$ (or, more generally $n \in \mathbb{Z}$ if $|n|<m$ ) we have

$$
N_{\mathrm{c}}=m+n, \quad k=m \quad \text { and } \quad N_{\mathrm{f}}=m+2 n,
$$

because then $Z(m+n, m+2 n, m)=e^{i \pi \phi_{\mathrm{GK}}} Z(m+n, m+2 n,-m)$. In addition, since the partition function on the r.h.s. is the complex conjugate of the one in the l.h.s., if we write $Z(m+n, m+2 n, m)=r e^{\mathrm{i} \theta}$ in polar form, we see that for partition functions characterized by (3.4) it holds that $\theta=\phi_{\mathrm{GK}} / 2$.

\section{Giveon-Kutasov duality}

The Giveon-Kutasov duality is between $\mathrm{U}\left(N_{\mathrm{c}}\right)$ and $\mathrm{U}\left(|k|+N_{\mathrm{f}}-N_{\mathrm{c}}\right)$, where $k$ is the ChernSimons level. In particular, the theories are:

- $\mathcal{N}=2 \mathrm{U}\left(N_{\mathrm{c}}\right)$ gauge theory with $N_{\mathrm{f}}$ flavors and a Chern-Simons term at level $k$.

- $\mathcal{N}=2 \mathrm{U}\left(|k|+N_{\mathrm{f}}-N_{\mathrm{c}}\right)$ gauge theory with $N_{\mathrm{f}}$ flavors and a Chern-Simons term at level $-k$. In addition, there are $N_{\mathrm{f}}^{2}$ uncharged chiral multiplets $M_{a}^{b}$, which couple through a superpotential $\tilde{q}^{a} M_{a}^{b} q_{b}$.

As explained in the Introduction, the Giveon-Kutasov duality specifically implies for the partition function

$$
Z_{N_{\mathrm{f}}, k}^{\mathrm{U}\left(N_{\mathrm{c}}\right)}(\eta)=e^{\operatorname{sgn}(k) \pi \mathrm{i}\left(c_{|k|, N_{\mathrm{f}}}-\eta^{2}\right)} Z_{N_{\mathrm{f}},-k}^{\mathrm{U}\left(|k|+N_{\mathrm{f}}-N_{\mathrm{c}}\right)}(-\eta),
$$

where $c\left(|k|, N_{\mathrm{c}}, N_{\mathrm{f}}\right)$ is a polynomial quadratic in the level $k$ (or rather on its absolute value) and the coefficients have a non-trivial dependence on $N_{\mathrm{f}}$ (and, we find, on $N_{\mathrm{c}}$ as well). As discussed in [28], this phase can be attributed to certain contact terms that must be added to the action to ensure reflection positivity. Thus, using the matrix model representation of the partition function of the $\mathcal{N}=3, \mathrm{U}\left(N_{\mathrm{c}}\right)$ theory with $N_{\mathrm{f}}$ fundamental flavors ${ }^{6}[22]$

$$
Z_{k, N_{\mathrm{f}}, N_{\mathrm{c}}}\left(\eta, m_{a}\right)=e^{\mathrm{i} \delta\left(N_{\mathrm{c}}, k, N_{\mathrm{f}} ; \eta, m_{a}\right)} \frac{1}{N_{\mathrm{c}} !} \int d^{N_{\mathrm{c}}} \lambda \prod_{j=1}^{N_{\mathrm{c}}} \frac{e^{-k \pi \mathrm{i} \lambda_{j}{ }^{2}+2 \pi \mathrm{i} \eta \lambda_{j}}}{\prod_{a=1}^{N_{\mathrm{f}}} 2 \cosh \pi\left(\lambda_{j}+m_{a}\right)} \prod_{i<j}\left(2 \sinh \pi\left(\lambda_{i}-\lambda_{j}\right)\right)^{2},
$$

where, following the notation and presentation in [22], $\delta$ is chosen so that $Z$ is real and positive, then the statement (4.1) is encapsulated in

$$
Z_{k, N_{\mathrm{f}}, N_{\mathrm{c}}}\left(\eta, m_{a}\right)=Z_{-k, N_{\mathrm{f}},|k|+N_{\mathrm{f}}-N_{\mathrm{c}}}\left(-\eta, m_{a}\right) .
$$

In [22], it is argued that an explicit formula for the relative phase can be computed by studying the contact terms of the dual theories [28] and the expression

$$
\begin{aligned}
\gamma\left(N_{\mathrm{c}}, k, N_{\mathrm{f}} ; \eta, m_{a}\right) & :=\delta\left(|k|+N_{\mathrm{f}}-N_{\mathrm{c}},-k, N_{\mathrm{f}} ;-\eta, m_{a}\right)-\delta\left(N_{\mathrm{c}}, k, N_{\mathrm{f}} ; \eta, m_{a}\right) \\
& =\frac{1}{24}\left(k^{2}+3\left(k+N_{\mathrm{f}}\right)\left(N_{\mathrm{f}}-2\right)+2\right)+\frac{1}{2} \eta^{2}-\frac{1}{2} k \sum_{a} m_{a}{ }^{2}-\eta \sum_{a} m_{a}
\end{aligned}
$$

\footnotetext{
${ }^{6}$ Setting $\mu_{i}=2 \pi \lambda_{i}$ and $m_{a}=m$ for all $a=1,2, \ldots, N_{\mathrm{f}}$ one obtains (1.3).
} 
is provided. Its derivation appeared in the previous work [21]. The formulas in [21] and [22] are very similar with the exception of a global $-2 \pi$ multiplicative re-scaling, which indeed seems to be missing in (4.2)-(4.4). Additionally, the expression in [21] does not contain the last term in (4.4), involving both the FI parameter and the masses. ${ }^{7}$ Former work of the same authors conjectured that [20]

$$
Z_{k, N_{\mathrm{f}}}^{\left(N_{\mathrm{c}}\right)}\left(\eta ; m_{a}\right)=e^{\operatorname{sgn}(k) \pi \mathrm{i}\left(c_{|k|, N_{\mathrm{f}}}-\eta^{2}\right)} e^{\sum_{a}\left(k \pi \mathrm{i} m_{a}^{2}+2 \pi \mathrm{i} \eta m_{a}\right)} Z_{-k, N_{\mathrm{f}}}^{\left(|k|+N_{\mathrm{f}}-N_{\mathrm{c}}\right)}\left(-\eta ; m_{a}\right),
$$

where

$$
c_{k, N_{\mathrm{f}}}=-\frac{1}{12}\left(k^{2}+3\left(N_{\mathrm{f}}-2\right) k+a_{N_{\mathrm{f}}}\right)
$$

with

$$
a_{N_{\mathrm{f}}}= \begin{cases}-1, & N_{\mathrm{f}}=1, \quad(\bmod 4) \\ 2, & N_{\mathrm{f}}=2,4 \quad(\bmod 4), \\ -13, & N_{\mathrm{f}}=3 \quad(\bmod 4) .\end{cases}
$$

It is mentioned in [21] that there is consistency between the two formulas, but in general they do give different results for the phase factor. For example, while the two formulas agree for $N_{\mathrm{f}}=1$ and $N_{\mathrm{f}}=2$ and generic $k$, they differ for $N_{\mathrm{f}}=3$. For instance

$$
-2 \pi \gamma\left(N_{\mathrm{c}}, 1,3, \eta=0, m_{a}=0\right)=-\frac{5}{4} \pi \quad \text { whereas } \quad \pi c_{1,3}=\frac{3}{4} \pi .
$$

Note also that the factor $\operatorname{sgn}(k)$ that appears in (4.5) but not in the other two, above mentioned, expressions, guarantees consistency if one applies the duality again on the r.h.s. of (4.5).

We further test the duality, in the $\mathcal{N}=3$ setting, of the matrix model (4.2) using the formalism developed in section 2 .

\subsection{Explicit expression for the phase factor}

For $N_{\mathrm{f}}>1$, it becomes computational intractable to estimate (1.3) in pen and paper. Thus, we programmed (1.3), using Mathematica, as a function which takes as input parameters the variables $\left(k, N_{\mathrm{f}}, N_{\mathrm{c}}, m, \eta\right)$. These computations are symbolical and work well for low values of $N_{\mathrm{f}}, N_{\mathrm{c}}, k$, however for larger values, the symbolical calculations become time consuming. ${ }^{8}$ As a first step, we solved symbolically (1.3) for low values $N_{\mathrm{f}}, N_{\mathrm{c}}$, see also section 3. We verified that neither the mass term nor the FI term couple with $k$, as expected and suggested in (4.4) and (4.5). Therefore, to further investigate the form of the quadratic function in $k$ we focus on the massless and $\eta=0$ case. This is convenient because floatingpoint arithmetic and parallelization methods on Mathematica scripts, speed up our code and enable us to compute cases up to $N_{\mathrm{f}}=12$.

\footnotetext{
${ }^{7}$ The duality considered in [21] is between $Z_{k, N_{\mathrm{f}}, N_{\mathrm{c}}}(\eta, m)$ and $Z_{-k, N_{\mathrm{f}},|k|+N_{\mathrm{f}}-N_{\mathrm{c}}}(\eta,-m)$ instead of eq. (4.3).

${ }^{8}$ All computations were performed on a laptop with Intel Core2 Duo CPU T6400 2.00 GHz and 3GB RAM and a Fujitsu Server Primergy TX100 S3 with 8 processors and 8GB RAM.
} 
Typically, our process is the following: for specific values of $N_{\mathrm{f}}$ and $N_{\mathrm{c}}$ we find the ratios

$$
\frac{Z_{N_{\mathrm{f}}, k}^{\mathrm{U}\left(N_{\mathrm{c}}\right)}(0)}{Z_{N_{\mathrm{f}},-k}^{\mathrm{U}\left(|k|+1 N_{\mathrm{f}}-N_{\mathrm{c}}\right)}(0)}=e^{\mathrm{i} \pi \theta}
$$

for $0<|k| \leq 5$. The ratio is always a phase, e.g. $e^{\mathrm{i} \pi \theta}, \theta \in \mathbb{R}$. We then determine a quadratic function in $|k|, \phi_{N_{\mathrm{f}}, N_{\mathrm{c}}}(k)$, that captures all phases for $0<|k| \leq 5$. We repeat for different values of $N_{\mathrm{f}}$ and $N_{\mathrm{c}}$ and certain patterns for the quadratic functions $\phi_{N_{\mathrm{f}}, N_{\mathrm{c}}}(|k|)$ arise.

We summarize our results in table 1 , where we present the parameter $\theta$ for different values of $N_{\mathrm{f}}, N_{\mathrm{c}}$ and $0<|k| \leq 5$. We observe the anti-symmetry between negative and positive $k$, which leads us to write $e^{i \pi \theta}=e^{\operatorname{sgn}(k) i \pi \phi_{N_{\mathrm{f}}, N_{\mathrm{c}}}(|k|)}$. This confirms the phase in (4.1) when $\eta=0$ and $m=0$. First, we see that neither (4.4) nor (4.6) are good candidates for reproducing the values in table 1. In particular, applying (4.4) and (4.5) with (4.6) for $N_{\mathrm{f}}=1$ and $k=1,2, \ldots, 5$ one finds

$$
\theta= \begin{cases}\frac{1}{4}, & k=1, \\ \frac{1}{4}, & k=2, \\ \frac{1}{12}, & k=3, \\ -\frac{1}{4}, & k=4, \\ -\frac{3}{4}, & k=5,\end{cases}
$$

respectively. These values are different from those presented in table 1 . Therefore we need to go beyond the existing conjectured quadratic functions and find a new one. Thus, we search for a universal quadratic function

$$
\phi\left(N_{\mathrm{f}}, N_{\mathrm{c}},|k|\right)=\alpha\left(N_{\mathrm{f}}, N_{\mathrm{c}}\right) k^{2}+\beta\left(N_{\mathrm{f}}, N_{\mathrm{c}}\right)|k|+\gamma\left(N_{\mathrm{f}}, N_{\mathrm{c}}\right),
$$

which captures all the values obtained. This is done in two steps. In the first step, for each $N_{\mathrm{f}}$ and $N_{\mathrm{c}}$ we use the $\theta$ values for $k=1,2,3$ to find a quadratic function. That is, we solve the system of equations

$$
\begin{aligned}
& \theta_{1}=\alpha_{N_{\mathrm{f}}, N_{\mathrm{c}}}+\beta_{N_{\mathrm{f}}, N_{\mathrm{c}}}+\gamma_{N_{\mathrm{f}}, N_{\mathrm{c}}} \\
& \theta_{2}=4 \alpha_{N_{\mathrm{f}}, N_{\mathrm{c}}}+2 \beta_{N_{\mathrm{f}}, N_{\mathrm{c}}}+\gamma_{N_{\mathrm{f}}, N_{\mathrm{c}}} \\
& \theta_{3}=9 \alpha_{N_{\mathrm{f}}, N_{\mathrm{c}}}+3 \beta_{N_{\mathrm{f}}, N_{\mathrm{c}}}+\gamma_{N_{\mathrm{f}}, N_{\mathrm{c}}}
\end{aligned}
$$

to find the parameters $\alpha_{N_{\mathrm{f}}, N_{\mathrm{c}}}, \beta_{N_{\mathrm{f}}, N_{\mathrm{c}}}, \gamma_{N_{\mathrm{f}}, N_{\mathrm{c}}}$. It is worth mentioning that there is not a unique quadratic function that gives rise to the same phase. Had we solved for $k=$ 2,3,4 we would have found different parameters which still give the same overall phase. Therefore, we find different quadratic functions for different values of $N_{\mathrm{f}}, N_{\mathrm{c}}$. For example, for $N_{\mathrm{f}}=N_{\mathrm{c}}=1$ we find $\phi_{1,1}(k)=\frac{5}{12} k^{2}-\frac{9}{4} k+\frac{25}{12}$ which gives $e^{\operatorname{sgn}(k) \pi \mathrm{i} \phi_{1,1}(|k|)} / e^{\pi \mathrm{i} \theta}=1$ for all $\theta$ in the first row of table 1 . We further do some "blind" tests computing $\theta$ for a $k>|5|$ and verifying the correctness of the expression $\phi_{N_{\mathrm{f}}, N_{\mathrm{c}}}(k)$. 


\begin{tabular}{|c|c|c|c|c|c|c|c|c|c|c|c|}
\hline & & \multicolumn{10}{|c|}{$k$} \\
\hline$N_{\mathrm{f}}$ & $N_{\mathrm{c}}$ & -5 & -4 & -3 & -2 & -1 & 1 & 2 & 3 & 4 & 5 \\
\hline 1 & 1 & $3 / 4$ & $1 / 4$ & $1 / 12$ & $3 / 4$ & $-1 / 4$ & $1 / 4$ & $-3 / 4$ & $-11 / 12$ & $-1 / 4$ & $-3 / 4$ \\
\hline 1 & 2 & $1 / 4$ & $1 / 4$ & $1 / 12$ & $3 / 4$ & $3 / 4$ & $-3 / 4$ & $-3 / 4$ & $1 / 12$ & $-1 / 4$ & $1 / 4$ \\
\hline 1 & 3 & $3 / 4$ & $1 / 4$ & $1 / 12$ & $3 / 4$ & $\mathrm{NaN}$ & $\mathrm{NaN}$ & $-3 / 4$ & $-11 / 12$ & $-1 / 4$ & $-3 / 4$ \\
\hline 2 & 1 & $3 / 4$ & $-1 / 2$ & $1 / 12$ & $-1 / 2$ & $-3 / 4$ & $3 / 4$ & $1 / 2$ & $-11 / 12$ & $1 / 2$ & $3 / 4$ \\
\hline 2 & 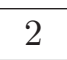 & $-3 / 4$ & $1 / 2$ & $1 / 12$ & $1 / 2$ & $-3 / 4$ & $3 / 4$ & $-1 / 2$ & $-11 / 12$ & $-1 / 2$ & $3 / 4$ \\
\hline 2 & 3 & $-3 / 4$ & $1 / 2$ & 12 & $1 / 2$ & $-3 / 4$ & $3 / 4$ & $1 / 2$ & 12 & $1 / 2$ & $3 / 4$ \\
\hline 2 & 4 & $-3 / 4$ & $1 / 2$ & $11 / 12$ & $1 / 2$ & $\mathrm{NaN}$ & $\mathrm{NaN}$ & $-1 / 2$ & $-11 / 12$ & $-1 / 2$ & $3 / 4$ \\
\hline 3 & 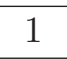 & $-3 / 4$ & $1 / 4$ & $5 / 12$ & $1 / 4$ & $1 / 4$ & $-1 / 4$ & $1 / 4$ & $-5 / 12$ & $-1 / 4$ & $3 / 4$ \\
\hline 3 & 2 & $1 / 4$ & $1 / 4$ & $-7 / 12$ & $-1 / 4$ & $-3 / 4$ & $3 / 4$ & $1 / 4$ & $7 / 12$ & $-1 / 4$ & $-1 / 4$ \\
\hline 3 & 3 & $-3 / 4$ & $1 / 4$ & $5 / 12$ & $1 / 4$ & $1 / 4$ & $-1 / 4$ & $1 / 4$ & $-5 / 12$ & $-1 / 4$ & $3 / 4$ \\
\hline 3 & 4 & $1 / 4$ & $1 / 4$ & $-7 / 12$ & $-1 / 4$ & $-3 / 4$ & $3 / 4$ & $1 / 4$ & $7 / 12$ & $-1 / 4$ & $-1 / 4$ \\
\hline 3 & 5 & $-3 / 4$ & $1 / 4$ & $5 / 12$ & $-1 / 4$ & $\mathrm{NaN}$ & $\mathrm{NaN}$ & $1 / 4$ & $-5 / 12$ & $-1 / 4$ & $3 / 4$ \\
\hline 4 & 1 & $3 / 4$ & $1 / 2$ & $-7 / 12$ & $-1 / 2$ & $3 / 4$ & $-3 / 4$ & $1 / 2$ & $7 / 12$ & $-1 / 2$ & $-3 / 4$ \\
\hline 4 & 2 & $3 / 4$ & $-1 / 2$ & $-7 / 12$ & $1 / 2$ & $3 / 4$ & $-3 / 4$ & $-1 / 2$ & $7 / 12$ & $1 / 2$ & $-3 / 4$ \\
\hline 4 & 3 & $3 / 4$ & $1 / 2$ & $-7 / 12$ & $-1 / 2$ & $3 / 4$ & $-3 / 4$ & $1 / 2$ & $7 / 12$ & $-1 / 2$ & $-3 / 4$ \\
\hline 4 & 4 & $3 / 4$ & $-1 / 2$ & $-7 / 12$ & $1 / 2$ & $3 / 4$ & $-3 / 4$ & $-1 / 2$ & $7 / 12$ & $1 / 2$ & $-3 / 4$ \\
\hline 4 & 5 & $3 / 4$ & & $-7 / 12$ & $1 / 2$ & 4 & $-3 / 4$ & $1 / 2$ & 71 & $1 / 2$ & $-3 / 4$ \\
\hline 5 & 1 & $-1 / 4$ & $1 / 4$ & $-1 / 12$ & $3 / 4$ & $3 / 4$ & $-3 / 4$ & $-3 / 4$ & $1 / 12$ & $-1 / 4$ & $1 / 4$ \\
\hline 5 & 2 & $3 / 4$ & & $11 / 12$ & $3 / 4$ & $-1 / 4$ & $1 / 4$ & $-3 / 4$ & $-11 / 12$ & $-1 / 4$ & $-3 / 4$ \\
\hline 5 & 3 & $-1 / 4$ & $1 / 4$ & $-1 / 12$ & $3 / 4$ & $3 / 4$ & $-3 / 4$ & $-3 / 4$ & $1 / 12$ & $-1 / 4$ & $1 / 4$ \\
\hline 5 & 4 & $3 / 4$ & $1 / 4$ & $11 / 12$ & $3 / 4$ & $-1 / 4$ & $1 / 4$ & $-3 / 4$ & $-11 / 12$ & $-1 / 4$ & $-3 / 4$ \\
\hline 5 & 5 & $-1 / 4$ & $1 / 4$ & $-1 / 12$ & $3 / 4$ & $3 / 4$ & $-3 / 4$ & $-3 / 4$ & $1 / 12$ & $-1 / 4$ & $1 / 4$ \\
\hline 6 & 1 & $1 / 4$ & $-1 / 2$ & $-1 / 12$ & $-1 / 2$ & $1 / 4$ & $-1 / 4$ & $1 / 2$ & $1 / 12$ & $1 / 2$ & $-1 / 4$ \\
\hline 6 & 2 & $1 / 4$ & $1 / 2$ & $-1 / 12$ & $1 / 2$ & $1 / 4$ & $-1 / 4$ & $-1 / 2$ & $1 / 12$ & $-1 / 2$ & $-1 / 4$ \\
\hline 6 & 3 & $1 / 4$ & $-1 / 2$ & $-1 / 12$ & $-1 / 2$ & $1 / 4$ & $-1 / 4$ & $1 / 2$ & $1 / 12$ & $1 / 2$ & $-1 / 4$ \\
\hline 6 & 4 & $1 / 4$ & $1 / 2$ & $-1 / 12$ & $1 / 2$ & $1 / 4$ & $-1 / 4$ & $-1 / 2$ & $1 / 12$ & $-1 / 2$ & $-1 / 4$ \\
\hline 6 & 5 & $1 / 4$ & $-1 / 2$ & $-1 / 12$ & $-1 / 2$ & $1 / 4$ & $-1 / 4$ & $1 / 2$ & $1 / 12$ & $1 / 2$ & $-1 / 4$ \\
\hline 7 & 1 & $1 / 4$ & $1 / 4$ & $-7 / 12$ & $-1 / 4$ & $-3 / 4$ & $3 / 4$ & $1 / 4$ & $7 / 12$ & $-1 / 4$ & $-1 / 4$ \\
\hline 8 & 1 & $-1 / 4$ & $1 / 2$ & $5 / 12$ & $-1 / 2$ & $-1 / 4$ & $1 / 4$ & $1 / 2$ & $-5 / 12$ & $-1 / 2$ & $1 / 4$ \\
\hline 9 & 1 & $3 / 4$ & $1 / 4$ & $11 / 12$ & $3 / 4$ & $-1 / 4$ & $1 / 4$ & $-3 / 4$ & $-11 / 12$ & $-1 / 4$ & $-3 / 4$ \\
\hline 11 & 1 & $-3 / 4$ & $1 / 4$ & $5 / 12$ & $-1 / 4$ & $1 / 4$ & $-1 / 4$ & $1 / 4$ & $-5 / 12$ & $-1 / 4$ & $3 / 4$ \\
\hline
\end{tabular}

Table 1. Values of the parameter $\theta$ for different values of $N_{\mathrm{f}}, N_{\mathrm{c}}$ and $k$ for the theory with $N_{\mathrm{f}}$ massless hypermultiplets, i.e. matrix model (1.3). NaN refers to instances where the dual theory is not well defined because the dual number of colours is negative, i.e. $|k|+N_{\mathrm{f}}-N_{\mathrm{c}}<0$, see discussion in section 3.3.

In the second step, we attempt to combine these different quadratic functions into a single quadratic function, such as (4.8). Our result is

$$
\phi\left(N_{\mathrm{f}}, N_{\mathrm{c}}, k\right)=\frac{1}{12}\left(5 k^{2}+\beta\left(N_{\mathrm{f}}, N_{\mathrm{c}}\right) k+\gamma\left(N_{\mathrm{f}}, N_{\mathrm{c}}\right)\right)
$$


where

$$
\begin{aligned}
& \beta\left(N_{\mathrm{f}}, N_{\mathrm{c}}\right)=(-1)^{N_{\mathrm{f}}} 3\left(N_{\mathrm{f}}+c+4\left(N_{\mathrm{c}}-1\right)\right), \\
& \gamma\left(N_{\mathrm{f}}, N_{\mathrm{c}}\right)=12\left(N_{\mathrm{f}}-1\right)\left(N_{\mathrm{c}}-1\right)+\left\{\begin{array}{lll}
-2, & N_{\mathrm{f}} & \bmod 4=2, \\
10, & N_{\mathrm{f}} & \bmod 4=0, \\
1, & N_{\mathrm{f}} & \bmod 4=1 \quad \text { or } \quad 3,
\end{array}\right.
\end{aligned}
$$

and

$$
c= \begin{cases}4, & N_{\mathrm{f}} \bmod 4=0 \\ 0, & \text { otherwise }\end{cases}
$$

The quadratic function (4.9) captures all the phases presented in the table 1, meaning that $e^{\operatorname{sgn}(k) \mathrm{i} \pi \phi\left(N_{\mathrm{f}}, N_{\mathrm{c}},|k|\right)} / e^{\mathrm{i} \pi \theta}=1$. This expression is further tested as follows. As explained above, in step 1 we have found a quadratic function for each $N_{\mathrm{f}}, N_{\mathrm{c}}$. This function is different from (4.9) and different for each $N_{\mathrm{f}}, N_{\mathrm{c}}$. Therefore, we further test the equality

$$
e^{\operatorname{sgn}(k) \mathrm{i} \pi \phi\left(N_{\mathrm{f}}, N_{\mathrm{c}},|k|\right)} / e^{\operatorname{sgn}(k) \mathrm{i} \pi \phi_{N_{\mathrm{f}}, N_{\mathrm{C}}}(|k|)}=1
$$

for $|k|>5$.

We do not claim that our result is the only valid quadratic function. It is the simplest one we could find for which $e^{\operatorname{sgn}(k) \mathrm{i} \pi \phi\left(N_{\mathrm{f}}, N_{\mathrm{c}},|k|\right)} / e^{\mathrm{i} \pi \theta}=1$. There might be other quadratic functions $\phi\left(N_{\mathrm{f}}, N_{\mathrm{c}}, k\right)$ which reproduce our results in table 1. As an open problem, it would be interesting to compare such results with a full computation coming from the complete analysis of supersymmetric Chern-Simons counterterms, since they characterize the anomaly [27, 28]. In [31] (see also [32]) all the required counterterms are explicitly given, actually for a much more general setting, including chiral theories, described by the matrix model (1.1). By taking the parameters $s_{1}, s_{2}$ in [31] as $s_{1}=s_{2}=N_{\mathrm{f}}$ one finds that the explicit expressions of the counterterms have a similar dependence, in appearance, in $N_{\mathrm{c}}$ and $N_{\mathrm{f}}$ to the one obtained here, although without the modular arithmetic (mod 4) behavior obtained here. The combination of the counterterms that give the phase factor is also well-known in general (see [31, eq. (5.13)] or [32, eq. (A.15)]), but we leave the eventual comparison of the results obtained here with matrix models with a direct explicit computation of the phase with the Chern-Simons counterterms [31, 32] as an open question for further work.

\subsection{Massive hypemultiplets and non-zero Fayet-Iliopoulos term}

Having determined the quadratic $k$-dependence of the phase factor we investigate the dependence on the mass, $m$, and the Fayet-Iliopoulos, $\eta$, terms in (1.3). As before, we use Mathematica to symbolically find the ratio

$$
\frac{Z_{N_{\mathrm{f}}, k}^{\mathrm{U}\left(N_{\mathrm{c}}\right)}(\eta)}{Z_{N_{\mathrm{f}},-k}^{\mathrm{U}\left(|k|+N_{\mathrm{f}}-N_{\mathrm{c}}\right)}(-\eta)}=e^{\mathrm{i} \pi\left(\operatorname{sgn}(k) \phi\left(N_{\mathrm{f}}, N_{\mathrm{c}},|k|\right)+\varphi\left(N_{\mathrm{f}}, N_{\mathrm{c}}, k, m, \eta\right)\right)} .
$$

To find this ratio we employ symbolical calculations using Mathematica. Alternatively, one could attempt to numerically find (4.11) for several values of $m$ and $\eta$ and then restore 


\begin{tabular}{|c|c|c|c|c|c|}
\hline & & \multicolumn{5}{|c|}{$k$} \\
\hline$N_{\mathrm{f}}$ & $N_{\mathrm{c}}$ & -2 & -1 & 1 & 2 \\
\hline 1 & 1 & $-\frac{i\left(m^{2}+2 m \pi \eta-2 \pi^{2} \eta^{2}\right)}{2 \pi}$ & $-\frac{i\left(m^{2}+4 m \pi \eta-4 \pi^{2} \eta^{2}\right)}{4 \pi}$ & $\frac{i\left(m^{2}-4 m \pi \eta-4 \pi^{2} \eta^{2}\right)}{4 \pi}$ & $\frac{i\left(m^{2}-2 m \pi \eta-2 \pi^{2} \eta^{2}\right)}{2 \pi}$ \\
\hline 1 & 2 & $-\frac{i\left(m^{2}+2 m \pi \eta-2 \pi^{2} \eta^{2}\right)}{2 \pi}$ & $-\frac{i\left(m^{2}+4 m \pi \eta-4 \pi^{2} \eta^{2}\right)}{4 \pi}$ & $\frac{i\left(m^{2}-4 m \pi \eta-4 \pi^{2} \eta^{2}\right)}{4 \pi}$ & $\frac{i\left(m^{2}-2 m \pi \eta-2 \pi^{2} \eta^{2}\right)}{2 \pi}$ \\
\hline 2 & 1 & & $-\frac{i\left(m^{2}+4 m \pi \eta-2 \pi^{2} \eta^{2}\right)}{2 \pi}$ & $\frac{i\left(m^{2}-4 m \pi \eta-2 \pi^{2} \eta^{2}\right)}{2 \pi}$ & \\
\hline 2 & 2 & $-\frac{i\left(m^{2}+2 m \pi \eta-\pi^{2} \eta^{2}\right)}{\pi}$ & $-\frac{i\left(m^{2}+4 m \pi \eta-2 \pi^{2} \eta^{2}\right)}{2 \pi}$ & $\frac{i\left(m^{2}-4 m \pi \eta-2 \pi^{2} \eta^{2}\right)}{2 \pi}$ & $\frac{i\left(m^{2}-2 m \pi \eta-\pi^{2} \eta^{2}\right)}{\pi}$ \\
\hline
\end{tabular}

Table 2. Dependence of the phase factor of the duality on the mass and FI terms for the theory with $N_{\mathrm{f}}$ massive hypermultiplets, i.e. the matrix model (1.3). For values of the parameters where the duality is not tested, due to computer memory limitations, the cell is left empty.

their functional dependence. The latter process is time consuming in many aspects and we focus on the symbolic approach. As we also mentioned previously, these calculations are very memory-demanding and one cannot handle as many cases as presented in table 1. However for the values of $N_{\mathrm{f}}, N_{\mathrm{c}}, k$ we compute, we do get a conclusive formula for the function $\varphi\left(N_{\mathrm{f}}, N_{\mathrm{c}}, k, m, \eta\right)$. In particular, in table 2 we present the term $\pi \varphi\left(N_{\mathrm{f}}, N_{\mathrm{c}}, k, m, \eta\right)$ in the right hand side of (4.11). Comparing the second row to the first and the fourth row to the third one, we observe that there is no $N_{\mathrm{c}}$ dependence in $\varphi$, hence $\varphi\left(N_{\mathrm{f}}, N_{\mathrm{c}}, k, m, \eta\right) \equiv$ $\varphi\left(N_{\mathrm{f}}, k, m, \eta\right)$. One can easily work out the following functional form of $\varphi$, which covers all the cases presented in table 2

$$
\begin{aligned}
\varphi\left(N_{\mathrm{f}}, k, m, \eta\right) & =\operatorname{sgn}(k)\left(\frac{|k| N_{\mathrm{f}} m^{2}}{4 \pi^{2}}-\frac{\operatorname{sgn}(k) N_{\mathrm{f}} m \eta}{\pi}-\eta^{2}\right) \\
& =\frac{k N_{\mathrm{f}} m^{2}}{4 \pi^{2}}-\frac{N_{\mathrm{f}} m \eta}{\pi}-\operatorname{sgn}(k) \eta^{2} .
\end{aligned}
$$

Recalling that the mass terms in (4.2) are related to the mass term in (1.3) via $2 \pi m_{a}=m$ we compare the (4.12) to the phase factors in (1.3) and notice that the two expressions are almost identical apart from a sign difference in the term $N_{\mathrm{f}} m \eta / \pi$.

While we found an expression for $\varphi$ we further test it for $\eta=0$, in which case we are able to explore more values of the parameters $N_{\mathrm{f}}, N_{\mathrm{c}}, k$. We present our findings in table 3 , which also provides further evidence for the functional form (4.12).

\section{3 $N_{\mathrm{f}}$ hypermultiplets with mass $m$ and $N_{\mathrm{f}}$ hypermultiplets with mass $-m$}

Next we present the results on the phase factor for the theory with $N_{\mathrm{f}}$ hypermultiplets of mass $m$ and $N_{\mathrm{f}}$ hypermultiplets of mass $-m$ discussed in section 2.1. In the case of $2 N_{\mathrm{f}}$ hypermultiplets, Giveon-Kutasov duality is between $\mathrm{U}\left(N_{\mathrm{c}}\right)$ and $\mathrm{U}\left(|k|+2 N_{\mathrm{f}}-N_{\mathrm{c}}\right)$, where $k$ is the Chern-Simons level. Similarly to the previous section, we implement in Mathematica the solution (2.14) with (2.15) and proceed in two steps. First we numerically and/or symbolically compute the phase factor for the massless and $\eta=0$ case. Then we turn on the mass terms and compute the mass dependence of the phase. For the first step, we 


\begin{tabular}{|c|c|c|c|c|c|c|c|}
\hline & & \multicolumn{5}{|c|}{$k$} \\
\hline$N_{\mathrm{f}}$ & $N_{\mathrm{c}}$ & -3 & -2 & -1 & 1 & 2 & 3 \\
\hline 1 & 1 & $-\frac{3 i m^{2}}{4 \pi}$ & $-\frac{i m^{2}}{2 \pi}$ & $-\frac{i m^{2}}{4 \pi}$ & $\frac{i m^{2}}{4 \pi}$ & $\frac{i m^{2}}{2 \pi}$ & $\frac{3 i m^{2}}{4 \pi}$ \\
\hline 1 & 2 & & $-\frac{i m^{2}}{2 \pi}$ & $-\frac{i m^{2}}{4 \pi}$ & $\frac{i m^{2}}{4 \pi}$ & $\frac{i m^{2}}{2 \pi}$ & \\
\hline 2 & 1 & & $-\frac{i m^{2}}{\pi}$ & $-\frac{i m^{2}}{2 \pi}$ & $\frac{i m^{2}}{2 \pi}$ & $\frac{i m^{2}}{\pi}$ & \\
\hline 2 & 2 & $\frac{3 i m^{2}}{2 \pi}$ & $-\frac{i m^{2}}{\pi}$ & $-\frac{i m^{2}}{2 \pi}$ & $\frac{i m^{2}}{2 \pi}$ & $\frac{i m^{2}}{\pi}$ & $\frac{3 i m^{2}}{2 \pi}$ \\
\hline 3 & 1 & & & $-\frac{3 i m^{2}}{4 \pi}$ & $\frac{3 i m^{2}}{4 \pi}$ & & \\
\hline 3 & 2 & & $-\frac{3 i m^{2}}{2 \pi}$ & $-\frac{3 i m^{2}}{4 \pi}$ & $\frac{3 i m^{2}}{4 \pi}$ & $\frac{3 i m^{2}}{2 \pi}$ & \\
\hline
\end{tabular}

Table 3. Dependence of the phase factor of the duality only on mass with $\eta=0$ for the theory with $N_{\mathrm{f}}$ massive hypermultiplets, see (1.3). For values of the parameters where the duality is not tested, due to computer memory limitations, the cell is left empty.

numerically compute the ratio

$$
\frac{\widetilde{Z}_{N_{\mathrm{f}}, k}^{\mathrm{U}\left(N_{\mathrm{c}}\right)}(0)}{\widetilde{Z}_{N_{\mathrm{f}},-k}^{\mathrm{U}\left(|k|+2 N_{\mathrm{f}}-N_{\mathrm{c}}\right)}(0)}=e^{\mathrm{i} \pi \theta}
$$

for $0<|k| \leq 5$. We present the values of the parameter $\theta$ in table 4 . One may proceed as before to find a universal expression as a function of $N_{\mathrm{f}}, N_{\mathrm{c}}, k$ that covers all the values in the table. However we observe that the values of $\theta$ for $N_{\mathrm{f}}=1,2,3,4$ in table 4 are identical to the values for $N_{\mathrm{f}}=2,4,6,8$ in table 1 . This is expected, because in the massless case the matrix models (1.3) and (1.4) are identical with $N_{\mathrm{f}}$ replaced by $2 N_{\mathrm{f}}$. Since we already have an expression for the quadratic function of the former matrix model, we use (4.9) replacing $N_{\mathrm{f}}$ with $2 N_{\mathrm{f}}$ and further test the remaining values of $N_{\mathrm{f}}$ and $N_{\mathrm{c}}$, confirming that $\phi\left(2 N_{\mathrm{f}}, N_{\mathrm{c}}, k\right)$ does give the expected results, which means that $e^{\mathrm{i} \pi \phi\left(2 N_{\mathrm{f}}, N_{\mathrm{c}}, k\right)} / e^{\mathrm{i} \pi \theta}=1$.

Having determined the quadratic $k$-dependence of the phase factor, we turn on the masses. We present the results for several cases of the parameters $N_{\mathrm{f}}, N_{\mathrm{c}}, k$ in table 5 . Comparing with the results in table 3 we observe that they differ by a factor of 2 , due to the fact that we now have two copies of $N_{\mathrm{f}}$ hypermultiplets. Therefore one may safely assume that (4.12) is still valid for $\eta=0$ and $N_{\mathrm{f}}$ replaced by $2 N_{\mathrm{f}}$.

The case of non-zero FI term will be examined in a more general setting of the theory, with $N_{\mathrm{f}}$ hypermultiplets of mass $m_{1}$ and $N_{\mathrm{f}}$ hypermultiplets of mass $m_{2}$, which is the topic of the next section.

\section{4 $N_{\mathrm{f}}$ hypermultiplets with mass $m_{1}$ and $N_{\mathrm{f}}$ hypermultiplets with mass $m_{2}$}

In this section we study a theory similar to (1.4) but with masses $m_{1}$ and $m_{2}$ and a non-zero FI term, $\eta$,

$$
\widehat{Z}_{N_{\mathrm{f}}}^{\mathrm{U}(N)}=\frac{1}{(2 \pi)^{N} N !} \int d^{N} \mu \frac{\prod_{i<j} 4 \sinh ^{2}\left(\frac{1}{2}\left(\mu_{i}-\mu_{j}\right)\right) e^{-\frac{1}{2 g} \sum_{i} \mu_{i}^{2}+\mathrm{i} \eta \sum_{i} \mu_{i}}}{\prod_{i}\left(4 \cosh \left(\frac{1}{2}\left(\mu_{i}+m_{1}\right)\right) \cosh \left(\frac{1}{2}\left(\mu_{i}+m_{2}\right)\right)\right)^{N_{\mathrm{f}}}} .
$$




\begin{tabular}{|c|c|c|c|c|c|c|c|c|c|c|c|}
\hline & & \multicolumn{10}{|c|}{$k$} \\
\hline$N_{\mathrm{f}}$ & $N_{\mathrm{c}}$ & -5 & -4 & -3 & -2 & -1 & 1 & 2 & 3 & 4 & 5 \\
\hline 1 & 1 & $-3 / 4$ & $-1 / 2$ & $11 / 12$ & $-1 / 2$ & $-3 / 4$ & $3 / 4$ & $1 / 2$ & $-11 / 12$ & $1 / 2$ & $3 / 4$ \\
\hline 1 & 2 & $-3 / 4$ & $1 / 2$ & $11 / 12$ & $1 / 2$ & $-3 / 4$ & $3 / 4$ & $-1 / 2$ & $-11 / 12$ & $-1 / 2$ & $3 / 4$ \\
\hline 1 & 3 & $-3 / 4$ & $-1 / 2$ & $11 / 12$ & $-1 / 2$ & $-3 / 4$ & $3 / 4$ & $1 / 2$ & $-11 / 12$ & $1 / 2$ & $3 / 4$ \\
\hline 1 & 4 & $-3 / 4$ & $1 / 2$ & $11 / 12$ & $1 / 2$ & NaN & NaN & $-1 / 2$ & $-11 / 12$ & $-1 / 2$ & $3 / 4$ \\
\hline 2 & 1 & $3 / 4$ & $1 / 2$ & $-7 / 12$ & $-1 / 2$ & $3 / 4$ & $-3 / 4$ & $1 / 2$ & $7 / 12$ & $-1 / 2$ & $-3 / 4$ \\
\hline 2 & 2 & $3 / 4$ & $-1 / 2$ & $-7 / 12$ & $1 / 2$ & $3 / 4$ & $-3 / 4$ & $-1 / 2$ & $7 / 12$ & $1 / 2$ & $-3 / 4$ \\
\hline 2 & 3 & $3 / 4$ & $1 / 2$ & $-7 / 12$ & $-1 / 2$ & $3 / 4$ & $-3 / 4$ & $1 / 2$ & $7 / 12$ & $-1 / 2$ & $-3 / 4$ \\
\hline 2 & 4 & $3 / 4$ & $-1 / 2$ & $-7 / 12$ & $1 / 2$ & $3 / 4$ & $-3 / 4$ & $-1 / 2$ & $7 / 12$ & $1 / 2$ & $-3 / 4$ \\
\hline 2 & 5 & $3 / 4$ & $1 / 2$ & $-7 / 12$ & $-1 / 2$ & $3 / 4$ & $-3 / 4$ & $1 / 2$ & $7 / 12$ & $-1 / 2$ & $-3 / 4$ \\
\hline 3 & 1 & $1 / 4$ & $-1 / 2$ & $-1 / 12$ & $-1 / 2$ & $1 / 4$ & $-1 / 4$ & $1 / 2$ & $1 / 12$ & $1 / 2$ & $-1 / 4$ \\
\hline 3 & 2 & $1 / 4$ & $1 / 2$ & $-1 / 12$ & $1 / 2$ & $1 / 4$ & $-1 / 4$ & $-1 / 2$ & $1 / 12$ & $-1 / 2$ & $-1 / 4$ \\
\hline 3 & 3 & $1 / 4$ & $-1 / 2$ & $-1 / 12$ & $-1 / 2$ & $1 / 4$ & $-1 / 4$ & $1 / 2$ & $1 / 12$ & $1 / 2$ & $-1 / 4$ \\
\hline 3 & 4 & $1 / 4$ & $1 / 2$ & $-1 / 12$ & $1 / 2$ & $1 / 4$ & $-1 / 4$ & $-1 / 2$ & $1 / 12$ & $-1 / 2$ & $-1 / 4$ \\
\hline 3 & 5 & $1 / 4$ & $-1 / 2$ & $-1 / 12$ & $-1 / 2$ & $1 / 4$ & $-1 / 4$ & $1 / 2$ & $1 / 12$ & $1 / 2$ & $-1 / 4$ \\
\hline 4 & 1 & $-1 / 4$ & $1 / 2$ & $5 / 12$ & $-1 / 2$ & $-1 / 4$ & $1 / 4$ & $1 / 2$ & $-5 / 12$ & $-1 / 2$ & $1 / 4$ \\
\hline 4 & 2 & $-1 / 4$ & $-1 / 2$ & $5 / 12$ & $1 / 2$ & $-1 / 4$ & $1 / 4$ & $-1 / 2$ & $-5 / 12$ & $1 / 2$ & $1 / 4$ \\
\hline 4 & 3 & $-1 / 4$ & $1 / 2$ & $5 / 12$ & $-1 / 2$ & $-1 / 4$ & $1 / 4$ & $1 / 2$ & $-5 / 12$ & $-1 / 2$ & $1 / 4$ \\
\hline 4 & 4 & $-1 / 4$ & $-1 / 2$ & $5 / 12$ & $1 / 2$ & $-1 / 4$ & $1 / 4$ & $-1 / 2$ & $-5 / 12$ & $1 / 2$ & $1 / 4$ \\
\hline 4 & 5 & $-1 / 4$ & $1 / 2$ & $5 / 12$ & $-1 / 2$ & $-1 / 4$ & $1 / 4$ & $1 / 2$ & $-5 / 12$ & $-1 / 2$ & $1 / 4$ \\
\hline 4 & 6 & $-1 / 4$ & $-1 / 2$ & $5 / 12$ & $1 / 2$ & $-1 / 4$ & $1 / 4$ & $-1 / 2$ & $-5 / 12$ & $1 / 2$ & $1 / 4$ \\
\hline 5 & 1 & $-3 / 4$ & $-1 / 2$ & $11 / 12$ & $-1 / 2$ & $-3 / 4$ & $3 / 4$ & $1 / 2$ & $-11 / 12$ & $1 / 2$ & $3 / 4$ \\
\hline 5 & 2 & $-3 / 4$ & $1 / 2$ & $11 / 12$ & $1 / 2$ & $-3 / 4$ & $3 / 4$ & $-1 / 2$ & $-11 / 12$ & $-1 / 2$ & $3 / 4$ \\
\hline 5 & 3 & $-3 / 4$ & $-1 / 2$ & $11 / 12$ & $-1 / 2$ & $-3 / 4$ & $3 / 4$ & $1 / 2$ & $-11 / 12$ & $1 / 2$ & $3 / 4$ \\
\hline 5 & 4 & $-3 / 4$ & $1 / 2$ & $11 / 12$ & $1 / 2$ & $-3 / 4$ & $3 / 4$ & $-1 / 2$ & $-11 / 12$ & $-1 / 2$ & $3 / 4$ \\
\hline 5 & 5 & $-3 / 4$ & $-1 / 2$ & $11 / 12$ & $-1 / 2$ & $-3 / 4$ & $3 / 4$ & $1 / 2$ & $-11 / 12$ & $1 / 2$ & $3 / 4$ \\
\hline 5 & 6 & $-3 / 4$ & $1 / 2$ & $11 / 12$ & $1 / 2$ & $-3 / 4$ & $3 / 4$ & $-1 / 2$ & $-11 / 12$ & $-1 / 2$ & $3 / 4$ \\
\hline 5 & 7 & $-3 / 4$ & $-1 / 2$ & $11 / 12$ & $-1 / 2$ & $-3 / 4$ & $3 / 4$ & $1 / 2$ & $-11 / 12$ & $1 / 2$ & $3 / 4$ \\
\hline
\end{tabular}

Table 4. Values of the parameter $\theta$ for different values of $N_{\mathrm{f}}, N_{\mathrm{c}}$ and $k$ of the theory with $2 N_{\mathrm{f}}$ massless hypermultiplets, actually the massless case of the matrix model (1.4). NaN refers to instances where the dual theory is not well defined because the dual number of colours is negative.

We solve it by substituting $z=c e^{\mu}$ and $c=e^{g\left(N-N_{\mathrm{f}}\right)}$ and following the steps in sections 2 and 2.1. We find

$$
\widehat{Z}_{N_{\mathrm{f}}}^{\mathrm{U}(N)}=e^{\frac{1}{2}\left(m_{1}+m_{2}\right) N N_{\mathrm{f}}} c^{-\frac{N}{2}\left(N+N_{\mathrm{f}}+2 i \eta\right)} \operatorname{det}\left(\left(h_{i}, h_{j}\right)\right)_{i, j=0}^{N-1},
$$

where the elements of the $\left(h_{i}, h_{j}\right)$ matrix are given by

$$
\begin{aligned}
\left(h_{i}, h_{j}\right)= & \frac{c^{i+j+1+\mathrm{i} \eta} e^{-\frac{1}{2 g}(\ln c)^{2}}}{\left(e^{m_{1}}-e^{m_{2}}\right)^{N_{\mathrm{f}}}} \sum_{s=0}^{N_{\mathrm{f}}-1} \frac{\left(\begin{array}{c}
N_{\mathrm{f}}+s-1 \\
s
\end{array}\right)}{\left(N_{\mathrm{f}}-s-1\right) !}\left(\frac{e^{m_{1}+m_{2}}}{e^{m_{1}}-e^{m_{2}}}\right)^{s} \\
& \times \sum_{n=0}^{N_{\mathrm{f}}-s-1}\left((-1)^{N_{\mathrm{f}}} C_{N_{\mathrm{f}}-s-1, n} I^{(n)}\left(\hat{\ell}+s, m_{1}\right)+(-1)^{s} C_{N_{\mathrm{f}}-s-1, n} I^{(n)}\left(\hat{\ell}+s, m_{2}\right)\right)
\end{aligned}
$$




\begin{tabular}{|c|c|c|c|c|c|c|c|}
\hline & & \multicolumn{6}{|c|}{$k$} \\
\hline$N_{\mathrm{f}}$ & $N_{\mathrm{c}}$ & -3 & -2 & -1 & 1 & 2 & 3 \\
\hline 1 & 1 & $-\frac{3 i m^{2}}{2 \pi}$ & $-\frac{i m^{2}}{\pi}$ & $-\frac{i m^{2}}{2 \pi}$ & $\frac{i m^{2}}{2 \pi}$ & $\frac{i m^{2}}{\pi}$ & $\frac{3 i m^{2}}{2 \pi}$ \\
\hline 1 & 2 & $-\frac{3 i m^{2}}{2 \pi}$ & $-\frac{i m^{2}}{\pi}$ & $-\frac{i m^{2}}{2 \pi}$ & $\frac{i m^{2}}{2 \pi}$ & $\frac{i m^{2}}{\pi}$ & $\frac{3 i m^{2}}{2 \pi}$ \\
\hline 2 & 1 & & & $-\frac{i m^{2}}{\pi}$ & & & \\
\hline 2 & 2 & & & $-\frac{i m^{2}}{\pi}$ & $\frac{i m^{2}}{\pi}$ & & \\
\hline 2 & 3 & & $-\frac{2 i m^{2}}{\pi}$ & $-\frac{i m^{2}}{\pi}$ & $\frac{i m^{2}}{\pi}$ & $\frac{2 i m^{2}}{\pi}$ & \\
\hline
\end{tabular}

Table 5. Dependence of the phase factor of the duality on mass for the theory with $N_{\mathrm{f}}$ hypermultiplets with masses $m$ and $N_{\mathrm{f}}$ hypermultiplets with masses $-m$. For values of the parameters where the duality is not tested, due to computer memory limitations, the cell is left empty.

where $\hat{\ell}=i+j+1-N+\mathrm{i} \eta$ and $C_{p, q}$ given by (2.5). One can verify that for $m_{1}=-m_{2}=m$ and $\eta=0$ finds the solution (2.15).

We again implement (4.16) in Mathematica and test the Giveon-Kutasov duality for low values of the parameters $N_{\mathrm{f}}, N_{\mathrm{c}}, k$. For $N_{\mathrm{f}}=N_{\mathrm{c}}=k=1$ we get

$$
\frac{\widehat{Z}_{N_{\mathrm{f}}}^{\mathrm{U}\left(N_{\mathrm{c}}\right)}}{\widehat{Z}_{N_{\mathrm{f}}}^{\mathrm{U}\left(|k|+2 N_{\mathrm{f}}-N_{\mathrm{c}}\right)}}=e^{\frac{3 \pi \mathrm{i}}{4}} e^{\frac{\mathrm{i}\left(m_{1}^{2}+m_{2}^{2}\right)}{4 \pi}-\mathrm{i} \eta\left(m_{1}+m_{2}\right)-\mathrm{i} \pi \eta^{2}} .
$$

For $N_{\mathrm{f}}=1, N_{\mathrm{c}}=2, k=1$ we find

$$
\frac{\widehat{Z}_{N_{\mathrm{f}}}^{\mathrm{U}\left(N_{\mathrm{c}}\right)}}{\widehat{Z}_{N_{\mathrm{f}}}^{\mathrm{U}\left(|k|+2 N_{\mathrm{f}}-N_{\mathrm{c}}\right)}}=e^{\frac{3 \pi \mathrm{i}}{4}} e^{\frac{\mathrm{i}\left(m_{1}^{2}+m_{2}^{2}\right)}{4 \pi}-\mathrm{i}\left(m_{1}+m_{2}\right) \eta-\mathrm{i} \pi \eta^{2}},
$$

whereas for $N_{\mathrm{f}}=1, N_{\mathrm{c}}=2, k=-2$ the ratio becomes

$$
\frac{\widehat{Z}_{N_{\mathrm{f}}}^{\mathrm{U}\left(N_{\mathrm{c}}\right)}}{\widehat{Z}_{N_{\mathrm{f}}}^{\mathrm{U}\left(|k|+2 N_{\mathrm{f}}-N_{\mathrm{c}}\right)}}=e^{\frac{\pi \mathrm{i}}{2}} e^{-\frac{\mathrm{i}\left(m_{1}^{2}+m_{2}^{2}\right)}{2 \pi}-\mathrm{i}\left(m_{1}+m_{2}\right) \eta+\mathrm{i} \pi \eta^{2}} .
$$

From these few examples we do observe a pattern, as we notice that only the $k$-quadratic phase depends on $N_{\mathrm{c}}$ and not the mass and FI terms. The latter terms are validated through

$$
\widehat{\varphi}\left(N_{\mathrm{f}}, k, m_{1}, m_{2}, \eta\right)=\frac{k N_{\mathrm{f}}\left(m_{1}^{2}+m_{2}^{2}\right)}{4 \pi^{2}}-\frac{N_{\mathrm{f}}\left(m_{1}+m_{2}\right) \eta}{\pi}-\operatorname{sgn}(k) \eta^{2},
$$

which is a generalization of (4.12) and is in agreement with the phase in (4.5), again up to a sign difference in the $\left(m_{1}+m_{2}\right) \eta$ term, as mentioned above.

\section{Acknowledgments}

We thank David García, Luis Melgar, Jorge Russo and Christian Vergu for comments and discussion. The work of MT is funded by an Investigador FCT position at Universidade 
de Lisboa (Reference IF/01767/2014). MT acknowledges former support at Universidad Complutense de Madrid, from MINECO (grant MTM2011-26912), Comunidad de Madrid (grant QUITEMAD+-CM, ref. S2013/ICE-2801) and the European CHIST-ERA project CQC (funded partially by MINECO grant PRI-PIMCHI-2011-1071).

Open Access. This article is distributed under the terms of the Creative Commons Attribution License (CC-BY 4.0), which permits any use, distribution and reproduction in any medium, provided the original author(s) and source are credited.

\section{References}

[1] V. Pestun, Localization of gauge theory on a four-sphere and supersymmetric Wilson loops, Commun. Math. Phys. 313 (2012) 71 [arXiv:0712.2824] [InSPIRE].

[2] V. Pestun, Localization for $\mathcal{N}=2$ Supersymmetric Gauge Theories in Four Dimensions, in New Dualities of Supersymmetric Gauge Theories, Mathematical Physics Studies, Springer International Publishing (2015) [arXiv: 1412.7134] [INSPIRE].

[3] K. Hosomichi, The localization principle in SUSY gauge theories, Prog. Theor. Exp. Phys. 2015 (2015) 11B101 [arXiv: 1502. 04543] [InSPIRE].

[4] S. Cremonesi, An Introduction to Localisation and Supersymmetry in Curved Space, PoS (Modave 2013) 002 [INSPIRE].

[5] P.J. Forrester, Log-gases and random matrices, Princeton University Press (2010).

[6] A. Kapustin, B. Willett and I. Yaakov, Exact Results for Wilson Loops in Superconformal Chern-Simons Theories with Matter, JHEP 03 (2010) 089 [arXiv:0909.4559] [INSPIRE].

[7] A. Kapustin, B. Willett and I. Yaakov, Nonperturbative Tests of Three-Dimensional Dualities, JHEP 10 (2010) 013 [arXiv: 1003.5694] [INSPIRE].

[8] D.L. Jafferis, The Exact Superconformal R-Symmetry Extremizes Z, JHEP 05 (2012) 159 [arXiv: 1012.3210] [INSPIRE].

[9] N. Hama, K. Hosomichi and S. Lee, Notes on SUSY Gauge Theories on Three-Sphere, JHEP 03 (2011) 127 [arXiv: 1012.3512] [INSPIRE].

[10] A. Barranco and J.G. Russo, Large- $N$ phase transitions in supersymmetric Chern-Simons theory with massive matter, JHEP 03 (2014) 012 [arXiv: 1401.3672] [INSPIRE].

[11] J.G. Russo, G.A. Silva and M. Tierz, Supersymmetric U(N) Chern-Simons-Matter Theory and Phase Transitions, Commun. Math. Phys. 338 (2015) 1411 [arXiv: 1407.4794] [INSPIRE].

[12] L.J. Mordell, The definite integral $\int_{-\infty}^{\infty} \frac{e^{a t^{2}+b t}}{e^{c t}+d} d t$ and the analytic theory of numbers, Acta Math. 61 (1933) 322.

[13] T. Eguchi and A. Taormina, On the Unitary Representations of $N=2$ and $N=4$ Superconformal Algebras, Phys. Lett. B 210 (1988) 125 [INSPIRE].

[14] T. Eguchi and Y. Sugawara, Non-holomorphic Modular Forms and SL(2, $\mathbb{R}) / \mathrm{U}(1)$ Superconformal Field Theory, JHEP 03 (2011) 107 [arXiv: 1012.5721] [INSPIRE].

[15] S. Zwegers, Mock Theta Functions, Ph.D. Thesis, Utrecht University (2002) [arXiv: 0807.4834]. 
[16] A. Folsom and K. Bringmann, Almost harmonic Maass forms and Kac-Wakimoto characters, J. Reine Angew. Math. 694 (2014) 179 [arXiv:1112.4726].

[17] A. Giveon and D. Kutasov, Seiberg Duality in Chern-Simons Theory, Nucl. Phys. B 812 (2009) 1 [arXiv:0808.0360] [InSPIRE].

[18] O. Aharony, IR duality in $D=3 N=2$ supersymmetric $\operatorname{USp}\left(2 N_{\mathrm{c}}\right)$ and $\mathrm{U}\left(N_{\mathrm{c}}\right)$ gauge theories, Phys. Lett. B 404 (1997) 71 [hep-th/9703215] [INSPIRE].

[19] K. Intriligator and N. Seiberg, Aspects of $3 d N=2$ Chern-Simons-Matter Theories, JHEP 07 (2013) 079 [arXiv: 1305.1633] [INSPIRE].

[20] A. Kapustin, B. Willett and I. Yaakov, Tests of Seiberg-like Duality in Three Dimensions, arXiv: 1012.4021 [INSPIRE].

[21] B. Willett and I. Yaakov, $N=2$ Dualities and $Z$ Extremization in Three Dimensions, arXiv: 1104.0487 [INSPIRE].

[22] A. Kapustin and B. Willett, Wilson loops in supersymmetric Chern-Simons-matter theories and duality, arXiv:1302.2164 [INSPIRE].

[23] X. Chen, Z.-C. Gu, Z.-X. Liu and X.-G. Wen, Symmetry protected topological orders and the group cohomology of their symmetry group, Phys. Rev. B 87 (2013) 155114 [arXiv:1106.4772] [INSPIRE].

[24] E. Witten, Fermion Path Integrals And Topological Phases, arXiv:1508.04715 [INSPIRE].

[25] L. Anderson and J.G. Russo, ABJM Theory with mass and FI deformations and Quantum Phase Transitions, JHEP 05 (2015) 064 [arXiv: 1502.06828] [INSPIRE].

[26] J.G. Russo and G.A. Silva, Exact partition function in $\mathrm{U}(2) \times \mathrm{U}(2)$ ABJM theory deformed by mass and Fayet-Iliopoulos terms, JHEP 12 (2015) 092 [arXiv:1510.02957] [INSPIRE].

[27] C. Closset, T.T. Dumitrescu, G. Festuccia, Z. Komargodski and N. Seiberg, Contact Terms, Unitarity and F-Maximization in Three-Dimensional Superconformal Theories, JHEP 10 (2012) 053 [arXiv:1205.4142] [INSPIRE].

[28] C. Closset, T.T. Dumitrescu, G. Festuccia, Z. Komargodski and N. Seiberg, Comments on Chern-Simons Contact Terms in Three Dimensions, JHEP 09 (2012) 091 [arXiv: 1206.5218] [INSPIRE].

[29] T. Morita and V. Niarchos, F-theorem, duality and SUSY breaking in one-adjoint Chern-Simons-Matter theories, Nucl. Phys. B 858 (2012) 84 [arXiv:1108.4963] [InSPIRE].

[30] D.S. Freed, Anomalies and Invertible Field Theories, Proc. Symp. Pure Math. 88 (2014) 25 [arXiv: 1404.7224] [INSPIRE].

[31] F. Benini, C. Closset and S. Cremonesi, Comments on 3d Seiberg-like dualities, JHEP 10 (2011) 075 [arXiv:1108.5373] [INSPIRE].

[32] A. Amariti and C. Klare, Chern-Simons and RG Flows: Contact with Dualities, JHEP 08 (2014) 144 [arXiv: 1405.2312] [INSPIRE]. 